Article

\title{
An Intelligent CFD-Based Optimization System for Fluid Machinery: Automotive Electronic Pump Case Application
}

\author{
Qiaorui Si ${ }^{1} \mathbb{D}$, Rong Lu ${ }^{1}$, Chunhao Shen ${ }^{1}$, Shuijing Xia ${ }^{2}$, Guochen Sheng ${ }^{1}$ and Jianping Yuan ${ }^{1, *}$ \\ 1 National Research Center of Pumps, Jiangsu University, Zhenjiang 212013, China; siqiaorui@ujs.edu.cn (Q.S.); \\ lurongdemail@163.com (R.L.); 18852854933@163.com (C.S.); shengguochenujs@163.com (G.S.) \\ 2 Pierburg Huayu Pump Technology Co. Ltd., Shanghai 201900, China; Shuijing.Xia@phptc.com \\ * Correspondence: yh@ujs.edu.cn; Tel.: +86-139-5280-1593
}

Received: 12 November 2019; Accepted: 31 December 2019; Published: 3 January 2020

check for updates

\begin{abstract}
Improving the efficiency of fluid machinery is an eternal topic, and the development of computational fluid dynamics (CFD) technology provides an opportunity to achieve optimal design in limited time. A multi-objective design process based on CFD and an intelligent optimization method is proposed in this study to improve the energy transfer efficiency, using the application of an automotive electronic pump as an example. Firstly, the three-dimensional CFD analysis of the prototype is carried out to understand the flow loss mechanism inside the pump and establish the numerical prediction model of pump performance. Secondly, an automatic optimization platform including fluid domain modeling, meshing, solving, post-processing, and design of experiment (DOE) is built based on three-dimensional parametric design method. Then, orthogonal experimental design and the multi-island genetic algorithm (MIGA) are utilized to drive the platform for improving the efficiency of the pump at three operating flowrates. Finally, the optimal impeller geometries are obtained within the limited $375 \mathrm{~h}$ and manufactured into a prototype for verification test. The results show that the highest efficiency of the pump increased by $4.2 \%$, which verify the effectiveness of the proposed method. Overall, the flow field has been improved significantly after optimization, which is the fundamental reason for performance improvement.
\end{abstract}

Keywords: automatic design; intelligent optimization method; CFD; fluid machinery; pumps

\section{Introduction}

Fluid machinery is an important energy conversion device, which is widely used in important sectors of the national economy such as hydropower, chemical processes, automobiles, nuclear power, and national defense [1,2]. With the deepening of energy saving and emission reduction, it is very important to improve the conveying efficiency of fluid machinery. Take the automobile industry as an example, the design of efficient cooling systems which are driven by blade pumps play an important role in the development of new energy vehicles. The former mechanical cooling water pump is mainly driven and coupled with the engine speed, which may either overcool or undercool. Electric water pumps are powered by adjustable speed motors and regulate operating conditions according to the cooling needs [3-5]. The pump unit coupled with the control program not only minimize the output power, but also meets the needs of the electric and intelligent development of the automotive industry. However, the efficiency of the pump is often below $40 \%$ due to unreasonable hydraulic design, leaving space for considerable energy savings. Moreover, pump geometries are mostly calculated based on ideal flow theory under a single flow rate using the traditional one-dimensional design method. However, the pump usually deviates from the optimal working condition, resulting in unstable flow 
phenomena such as secondary flow and flow separation inside the flow channel during the actual operation [6-8]. Thus, the study on the multi-objective optimization method of efficient pumps is also very important to the designers. However, there is still a lack of theoretical support and effective optimization tools for pump designing in the background of faster product updates.

Pump design theory has evolved from traditional one-dimensional design to three-dimensional design. With the rapid development of computer technology, the application of computational fluid dynamics (CFD) combined with optimization methods have become a popular and effective technique in turbomachinery design [9]. Wu et al. [10] investigated the effects of trailing edge modification on the performance of the mixed-flow pump through CFD analysis. Zhou et al. [11] compared the internal flow characteristics of a new kind of three-dimensional surface return diffuser to traditional ones using steady CFD simulation in order to improve the hydrodynamic performance of the deep-well centrifugal pump. Osman et al. [12] numerically investigated two multistage axially split centrifugal pumps with different channel designs between its stages, the flow losses were compared by entropy production. Wang et al. [13] established an energy loss model (ELM) to determine the relationships among the different types of energy losses in a multistage centrifugal pump, and a method was proposed to optimize the pump efficiency based on the ELM and CFD. The optimal design method of pumps has been studied extensively by many scholars. There are several methods to improve the pump performance, such as the empirical design method, approximate model method, and optimization algorithm. Liu et al. [14] implemented the orthogonal design with five factors and four levels to optimize a multiphase pump, the influence of each of the factors on the pressure rise was estimated, and the optimized ranges of these parameters were determined. The above method achieved some success but could not overcome the influence of human factors on the optimization results because it still relies on the empirical coefficients. In recent years, intelligent optimization algorithms have been developed rapidly, such as the genetic algorithm, ant colony algorithm, no-no search algorithm, simulated annealing algorithm, particle swarm algorithm, and so on [15-19]. With the optimization of pump performance, these intelligent algorithms are gradually adopted by pump researchers. Pei et al. [20] constructed an accurate nonlinear function between the optimization target and the pump design variables by utilizing an artificial neural network, and the modified particle swarm algorithm was further applied to optimize the mathematical model. Yuan et al. [21] adopted optimal Latin hypercube design, CFD simulation combined with the Kriging model were used to achieve the sample points for space-filling and establish the approximate optimization model, the best combination of impeller parameters were finally obtained by solving the approximation model with a genetic algorithm. Zhang et al. [22] proposed a multi-objective method to optimize a double suction centrifugal pump based on the Simulation Kriging Experiment (SKE) approach. Wang et al. [23] used radial basis function neural network combined with the Non-dominated Sorting Genetic Algorithm-II (NSGA-II) genetic algorithm to optimize a high-speed, mixed-flow pump. However, the optimization periods of these two methods are relatively short and it is difficult to obtain an overall optimal solution. In order to eliminate the deviation generated by the build of the approximation model, the researchers used the natural heuristic algorithm to optimize the performance globally. For example, Zhang et al. [24] utilized a genetic algorithm to optimize the geometry of a spiral axial-flow mixing pump. In recent years, a lot of intelligence algorithms have been proposed. Because there is no crossover and mutation process in the genetic algorithm, the convergence speed is greatly accelerated $[25,26]$. In summary, the pump optimization methods have undergone an improvement process from single target to multi-objective optimization, from experience to theory. Currently, with the advance of computer technology, automation, and artificial intelligence, automatic design methods without human intervention are being developed and applied in various fields.

This study focuses on the hydraulic optimization of automotive electronic pumps. Firstly, numerical simulations for a given model were carried out to build the prediction process of pump efficiency. Secondly, the target of this multi-objective optimization was established by weighting analysis. Then, an automatic optimization platform was established including parametric design, CFD and DOE. Orthogonal design, a kind of DOE method, was implemented in advance to estimate 
the influence of four selected factors on pump efficiency and head. The optimized ranges of these parameters were also determined. After, one kind of DOE namely the multi-island genetic algorithm (MIGA) was utilized to drive the platform for optimizing the weighted average efficiency under three operating flowrates. Finally, the optimized hydraulic scheme was obtained in a limited $375 \mathrm{~h}$ by using a normal desktop computer without running in parallel and was proven to improve the pump efficiency by $4.2 \%$ through the experiment. The proposed method may provide some theoretical guidance for hydraulic optimization of fluid machinery.

\section{Optimization Design Process and Modeling Method}

\subsection{Optimization Process}

In traditional design of fluid machinery, taking pumps for example, research procedures involve manufacturing prototypes, performance tests, results analyses, and optimal design. The design is mostly two-dimensional and relies on the experience of the designer. However, creating prototypes may cost a lot of time and money. Moreover, a large number of prototype tests would inevitably cause significant manufacturing and testing errors. The automatic design with the intelligent optimization method of fluid machinery presented in this study is based on the combination of CFD technology and optimization theory. An accurate CFD simulation method needs to be established first, which could provide virtual experimental results for optimization. Meanwhile, an automatic optimization platform including all CFD process such as fluid domain modeling, meshing, solving, post-processing, and design of experiment (DOE) was built for future optimization processes. The modeling of the pump geometry is based on the three-dimensional parametric design method, which means the fluid domain could be modified by DOE arrangements. The main parameters that influence the hydraulic performance of centrifugal pumps were screened out, and orthogonal design was applied to obtain the spacing-filling sample points. Significance test of optimization variables was conducted based on analysis of variance (ANOVA). Further, the MIGA optimization method was utilized to drive the platform for improving the efficiency of the pump automatically under three flowrates. Finally, an optimized scheme was obtained and the prototype was 3D printed to experimentally validate the optimal results. The whole optimization process is shown in Figure 1.

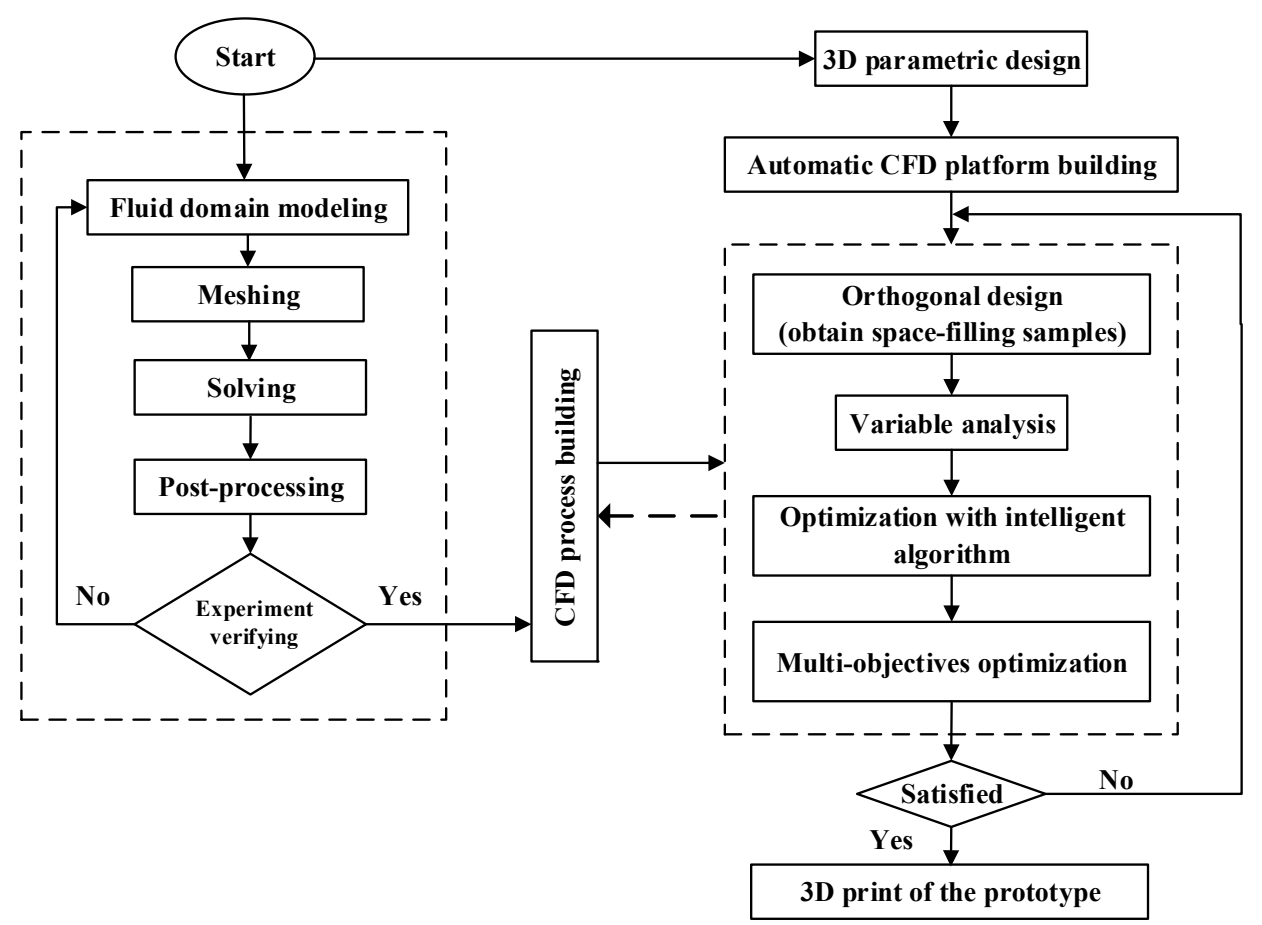

Figure 1. The flowchart of optimization process. CFD, computational fluid dynamics. 


\subsection{Pump Model}

The electronic pumps used in a car are always single-stage, single-suction centrifugal pumps equipped with a Direct Current (DC) brushless motor. They are characterized by simple structure, reliable operation, and convenient speed regulation. The motor contains the stator, the rotor, and shaft. The rotor will rotate after power-on under electromagnetic induction. Moreover, the electronic pump is equipped with a control unit that is connected to the driving computer. The rotational speed of the pump would be adjusted by pulse width modulation (PWM) controls according to heat dissipation of the cooling system, which may ensure the pump operates at a constant operation area after dimensionless processing [1].

\subsubsection{Pump Geometry and Losses Analysis}

The structure of automotive electronic pumps used in this study is shown in Figure 2a. The coolant liquid is sucked into the impeller, obtains pressure head after rotating with the impeller, and enters into the volute passage under centrifugal force. In this process, electrical energy is converted into the pressure energy of the fluid with a lot of losses, such as mechanical loss, leakage loss, disc friction losses, and hydraulic loss. Mechanical loss refers to the energy loss due to the mechanical friction in the pump, including the disk friction loss, bearing loss, and shaft seal loss. Leakage loss refers to the clearance leakage in the pump including the leakage loss at the front ring, rear ring, and balance holes. Hydraulic loss refers to the energy loss through the flow passage, including the inlet section, outlet section, pump cavity, impeller, and volute. The flow inside the pump passage is full 3D unsteady turbulence flow, including flow separation, backflow, circulation, instability flow, jet-wake flow, vortex, and even cavitation. As the most important part of energy transfer, the geometry of the impeller has a significant effect on hydraulic losses, pumping ability, and inherent reliability. Impeller-related losses dominate the pump efficiency. Therefore, the optimization of this study is mainly aimed at the impeller.

The pump performance characteristic curves at different rotational speeds are shown in Figure $2 b$. The pump would work at point $A$ in actual operation at design rotational speed $n_{\mathrm{d}}$ because of system resistance. Similarly, the pump would work at point $A_{1}$ in actual operation at rotational speed $n_{1}$ based on similarity law if Reynolds number changes within a certain range. The related losses of the pump under different rotational speeds also follows the similar law. Thus, it is equivalent to evaluate the pump performance only at the design rotational speed. The hydraulic parameters of the selected model pump are as follows: design rotational speed $n_{\mathrm{d}}$ is $5400 \mathrm{r} / \mathrm{min}$, design head $H_{\mathrm{d}}$ is $7.5 \mathrm{~m}$, design flow rate $Q_{\mathrm{d}}$ is $1.4 \mathrm{~m}^{3} / \mathrm{h}$. Table 1 provides a list of the main geometric parameters of the pump. Blade angle is relative to tangential direction. Considering the inlet pressure of the pump in the real system, this study does not involve cavitation problems.

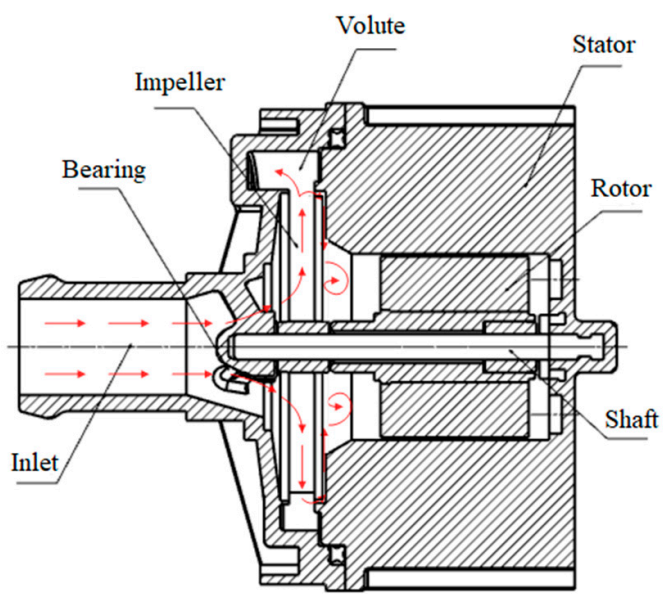

(a)

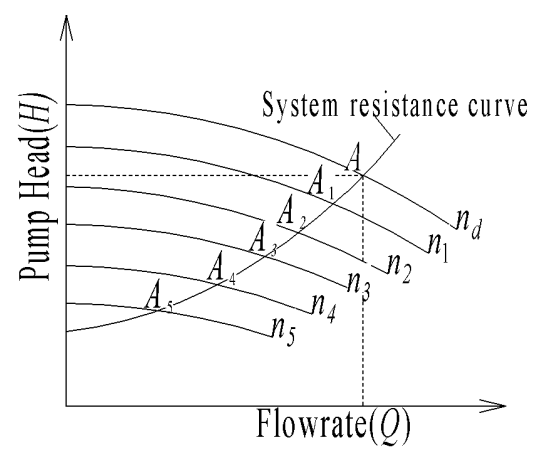

(b)

Figure 2. Automobile electronic pump model: (a) Structure diagram; (b) Performance characteristic curves. 
Table 1. Main geometric parameters of the pump.

\begin{tabular}{ccccc}
\hline Description & Parameter & Symbol & Unit & Value \\
\hline \multirow{4}{*}{ Impeller } & Inlet diameter & $D_{1}$ & $\mathrm{~mm}$ & 20 \\
& Outlet diameter & $D_{2}$ & $\mathrm{~mm}$ & 47 \\
& Outlet width & $b_{2}$ & $\mathrm{~mm}$ & 2.2 \\
& Blade inlet angle & $\beta_{1}$ & $\circ$ & 20 \\
& Blade number & $Z$ & - & 5 \\
& Blade outlet angle & $\beta_{2}$ & $\circ$ & 41 \\
\hline \multirow{3}{*}{ Volute } & Inlet diameter & $D_{3}$ & $\mathrm{~mm}$ & 50 \\
& Inlet width & $b_{3}$ & $\mathrm{~mm}$ & 6 \\
& Pipe diameter & $D$ & $\mathrm{~mm}$ & 17 \\
\hline
\end{tabular}

\subsubsection{Optimization Objectives}

The objective of the optimization is to build the relationships between the geometrical parameters of the impeller and the various kinds of energy losses in the pump, with the ultimate goal of minimizing the total energy loss. Thus, the following geometric parameters of impeller were selected as optimization variables based on the results of previous practice, such as the impeller outlet diameter $D_{2}$, blade outlet width $b_{2}$, blade number $Z$, blade outlet angle $\beta_{2}$, blade inlet angle $\beta_{1}$, leading edge tangential angle $t_{3}$, trailing edge tangential angle $\phi$, and blade thickness $\delta$. Moreover, the electronic water pump needs to operate under multiple working flowrates according to the working state of the cooling system. Therefore, a multi-point optimization is necessary. To broaden the high efficiency range of the model pump, the weighted average efficiency of three flow conditions, namely $0.8 Q_{d}, 1.0 Q_{d}, 1.25 Q_{d}$, were set as the objective function, and the design head was set as a constraint.

In general, the time of optimization with the intelligent method will be increased with both the number of parameters and the range of each parameter. In this study, exactly four parameters, impeller outlet diameter $D_{2}$, blade outlet width $b_{2}$, blade number $Z$, blade outlet angle $\beta_{2}$, were selected for the orthogonal experiment at the first step. These four parameters are considered to play most important roles in the comprehensive performance of centrifugal pumps $[6,13]$. Local optimization can be achieved, and then the range of parameters can be reduced in this step. However, because the optimization is also a multi-objective problem, it is necessary to establish the model with the highest weighted average efficiency under three working flowrates. The optimization problem can be formulated as:

Solve $\boldsymbol{X}=\left[D_{2}, b_{2}, Z, \beta_{2}\right]^{\mathrm{T}}$ make:

$$
\begin{gathered}
f(X)=\frac{\sum_{i=1}^{3} \omega_{i} \cdot \eta_{i}(\boldsymbol{X})}{\sum_{i=1}^{3} \omega_{i}} \rightarrow \max , \\
\text { subject to } \begin{array}{c}
\boldsymbol{X} \in \mathrm{R} \\
H_{\mathrm{d}} \geq 7.5 \mathrm{~m}
\end{array}
\end{gathered}
$$

where $X$ is a vector of all design variables, $R$ is the set of variable ranges, $\eta_{1}, \eta_{2}, \eta_{3}$ represent the efficiency of $0.8 Q_{d}, 1.0 Q_{d}$, and $1.25 Q_{d}$, respectively. Accordingly, $\omega_{i}$ represents the weighting factors.

Analytic hierarchy process (AHP) was developed in the late 1970s by Saaty [27]. Due to its simplicity, ease of use, and great flexibility, this technique has been widely used as a decision model to deal with multi-criteria evaluation. However, AHP does not take into account the inherent uncertainty and imprecision. Moreover, the comparison matrices used in AHP are combined with crisp scales. To deal with the uncertainty or vagueness of data, fuzzy analytic hierarchy process (FAHP) was utilized to derive the weight of every objective $[28,29]$. In FAHP, the importance of factor A to factor B is obtained and the fuzzy judgment matrix is generated after comparing different factors. This study use FAHP with the following steps. 
Firstly, build and construct a fuzzy complementary judgment matrix. The pairwise comparison among factors is made to determine the importance of one factor relative to the other factors, and then the judgment matrix is generated:

$$
\boldsymbol{A}=\left(a_{i j}\right)_{N \times N^{\prime}}
$$

where $a_{i j}$ represents the evaluation of the relative importance of the factor $i$ to the factor $j, a_{j i}=1 / a_{i j}$.

Secondly, calculate weight vector:

$$
\boldsymbol{\omega}_{i}^{*}=\left(b_{1}, b_{2}, \ldots, b_{N}\right)^{\mathrm{T}}
$$

where $i=1,2,3, \ldots, N ; b_{i}=\sqrt[N]{a_{i 1} \cdot a_{i 2} \ldots a_{i N}}$

Thirdly, calculate the normalized weight value for each weight vector obtained from Equation (4):

$$
\omega_{i}=b_{i} / \sum_{i=1}^{N} b_{i}
$$

Fourthly, sum the elements in each column:

$$
S_{i}=\left(\sum_{j=1}^{N} a_{1 j}, \sum_{j=1}^{N} a_{2 j}, \ldots \sum_{j=1}^{N} a_{N j}\right)^{\mathrm{T}} .
$$

Fifthly, calculate the maximum eigenvalue of the matrix. It would be used to calculate the following consistency index.

$$
\lambda_{\max }=\sum_{i=1}^{N} \boldsymbol{\Omega}_{i} \cdot S_{i}
$$

where $\boldsymbol{\Omega}_{i}=\left(\omega_{1}, \omega_{2}, \ldots, \omega_{N}\right)$

Sixthly, consistency test. A consistency ratio $(C R)$ is obtained in addition to the corresponding principal eigenvector when using FAHP for criteria weighting. It represents the priority vector which is integrated by the intended weights. The consistency ratio is used to measure if this eigenvector estimates the weight vector well. It is obtained by division of the consistency index $(C I)$ and the appropriate average random consistency index $(R I)$ :

$$
\begin{gathered}
C R=\frac{C I}{R I^{\prime}} \\
C I=\frac{\lambda_{\max }-N}{N-1},
\end{gathered}
$$

where $\lambda_{\max }$ is the maximum eigenvalue of the comparison matrix, and $N$ is the order of objective matrix. The value of the $R I$ is related to $N$, which can be obtained from Table 2. If $C R$ is greater than 0.1 , the comparison matrix is inconsistent and should be revised.

Table 2. Random consistency index $(R I)$.

\begin{tabular}{cccccccccccc}
\hline $\boldsymbol{N}$ & 1 & 2 & 3 & 4 & 5 & 6 & 7 & 8 & 9 & 10 & 11 \\
\hline $\boldsymbol{R} \boldsymbol{I}$ & 0 & 0 & 0.58 & 0.9 & 1.12 & 1.24 & 1.34 & 1.41 & 1.45 & 1.49 & 1.51 \\
\hline
\end{tabular}

The second step for the optimization is to use the intelligent method. The impeller parameters mentioned before, the impeller outlet diameter $D_{2}$, blade outlet width $b_{2}$, blade number $Z$, blade outlet angle $\beta_{2}$, blade inlet angle $\beta_{1}$, leading edge tangential angle $t_{3}$, trailing edge tangential angle $\phi$, and blade thickness $\delta$, are used for variable parameters to find the optimal combination at this step. Batch command is used to drive the following cycle of the numerical simulation process. 


\subsection{Numerical Method}

The key to intelligent optimization is to build a numerical calculation platform which runs automatically. So, the reliability of the numerical calculation process is particularly important.

\subsubsection{Establishing the Calculation Domain}

The hydraulic performance of the model pump is predicted by means of a CFD process in this study. The computational domain of the original pump is shown in Figure 3, which is comprised of four components, namely the inlet, impeller, pump chamber, and volute. In order to consider the full development of turbulence, the inlet and volute outlet pipes are properly extended. The inlet domain is directly connected to the impeller domain with a rotor-stator interface. The impeller domain is also directly connected to the chamber domain with a rotor-stator interface.

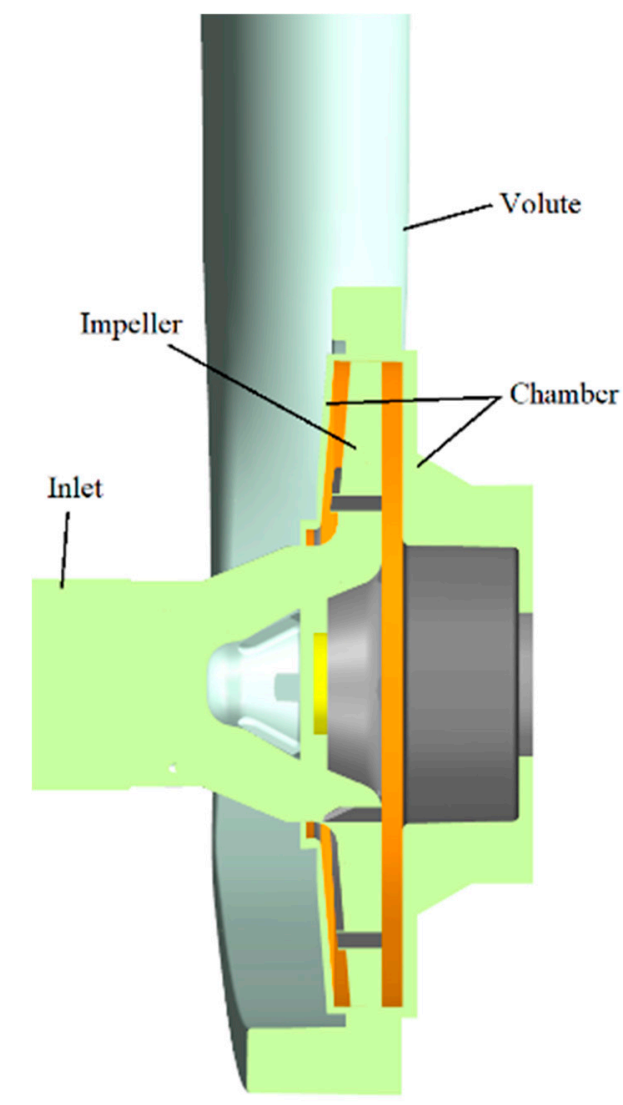

Figure 3. Model of computational domains.

\subsubsection{Mesh Sensitivity Analysis}

The calculation domains should be discretized by meshes before simulation. Due to the strong adaptability to complex geometry, tetrahedral unstructured grids generated by software Ansys ICEM14.1 are adopt in this study. The meshes of all computational flow domains are displayed in Figure 4. 


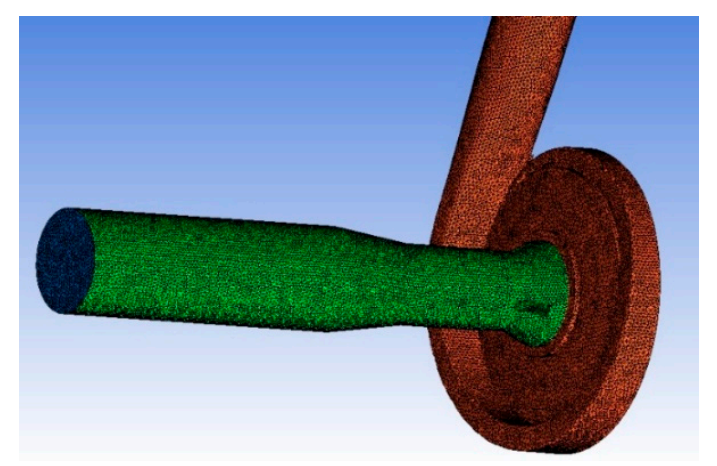

Figure 4. Computational meshes.

The number of mesh elements can significantly influence the simulation results. Seven sets of meshes are used for grid independence analysis, with elements rising from $34 \times 10^{4}$ to $251 \times 10^{4}$. Grid convergence study is done at design flowrate using the Reynolds-averaged Navier-Stokes equations (RANS) method and boundary conditions described below. The Reynolds number value is about $6.2 \times 10^{5}$ based on the formula $R_{\mathrm{e}}=u_{2} \cdot D_{2} / 2 v \cdot u_{2}$ represents the tangential velocity of the flow at the blade tip clearance under rated rotational speed $5400 \mathrm{r} / \mathrm{min} . v$ represents the kinematic viscosity of the fluid. So, it is reasonable to perform the following simulations in the framework of RANS. Seen from Figure 5, the overall difference of pump head is within $1 \%$ when the number of mesh elements exceed 1.68 million, which means the grid number has little effect on the calculation results. In order to balance the computational accuracy and the total calculation time, meshes with 168 million elements are employed for the following investigation.

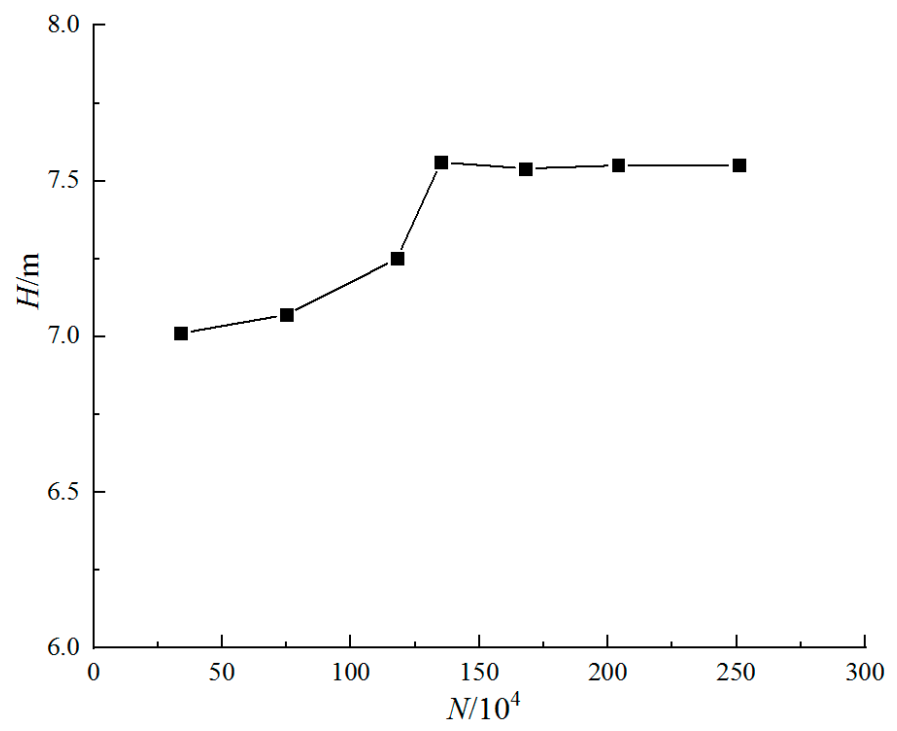

Figure 5. Analysis of grid independence.

\subsubsection{Governing Equations and Boundary Conditions}

The three-dimensional turbulent flow inside the pump is basically controlled by the law of mass and momentum conservation. For steady and incompressible flow, the control equations can be formulated as:

$$
\begin{gathered}
\nabla \cdot \vec{V}=0 \\
\frac{d \vec{V}}{d t}=\vec{F}-\frac{1}{\rho} \nabla p+v \nabla^{2} \vec{V},
\end{gathered}
$$


where $\vec{V}$ represents the velocity vector, $p$ represents pressure, $\vec{F}$ represents mass force, $v$ represents kinematic viscosity, $\rho$ represents the fluid density.

Due to different turbulence models influencing the calculation results of specific flow problems, selecting the suitable turbulence model can improve the calculation accuracy. In the present study, the fluid medium is set as water at $25^{\circ} \mathrm{C}$. The simulation is conducted by ANSYS CFX14.1 solver, which provides a number of turbulence models. Among the turbulence models, $k-\varepsilon$ and $k-\omega$ are known in engineering applications. The $\varepsilon$ equation of the standard $k-\varepsilon$ model must use a wall function to solve the terms, and the simulation results are not accurate in the case of severe flow separation. The standard $k-\omega$ model is improved for a low Reynolds number and shear flow. Moreover, the Shear Stress Transfer (SST) $k-\omega$ turbulence model uses a hybrid function which acts as a standard $k-\omega$ model on the near wall and the standard $k-\varepsilon$ model on the far wall area to correct the turbulent viscosity formula, while taking into account the turbulent shear stress. It has been proven to be more suitable for rotating machinery because it provides a better solution to the boundary layer [30]. The internal flow of the rotating pumps is three-dimensional turbulence flow, with strong flow separation, rotor-stator interaction, backflow, and so on. In addition, the electronic water pump has a high speed, and its flow losses mainly appear near the wall surface. Therefore, the SST $k-\omega$ turbulence model is adopted in this study to simulate the internal flow of the model pump based on the finite volume method.

The boundary conditions consist of an imposed stable total pressure with a turbulence intensity of $5 \%$ at the inlet and flow rates at outlet. All physical surfaces are set as no-slip walls. Considering the rotational characteristic, the rotating coordinate system is applied in impeller domain. The interfaces between the rotational domain and stationary ones are set as frozen rotor for steady simulation. The root mean square (RMS) residual error is used to judge whether the calculation is converged, setting values as $10^{-5}$.

\subsubsection{Experiment Validation}

According to the Bernoulli equation, the pump head $H$ is formulated as:

$$
H=\frac{p_{2}-p_{1}}{\rho g}+\frac{v_{2}^{2}-v_{1}^{2}}{2 g}+\left(z_{2}-z_{1}\right)
$$

where $p_{1}$ and $p_{2}$ imply the inlet static pressure and outlet static pressure respectively; $v_{1}$ and $v_{2}$ denote the average velocities of the inlet and outlet section respectively; $z_{1}$ and $z_{2}$ are the heights in vertical direction at the inlet and outlet of the model pump; $\rho$ denotes the fluid density, $997 \mathrm{~kg} / \mathrm{m}^{3} ; g$ denotes the local acceleration of gravity, $9.8 \mathrm{~m} / \mathrm{s}^{2}$.

The efficiency of centrifugal pump is the ratio of useful power to input power, defined as follows:

$$
\eta=\frac{\rho g Q H}{P_{e}}
$$

where $P_{e}$ denotes the shaft power calculated by the input power and motor efficiency. The way to obtain the value of $P_{e}$ in an experiment is different from the simulation because the torque $T$ acting on the rotor is easy to obtain in CFD. $P_{e}$ is equal to $n_{\mathrm{d}} \cdot T / 9550$.

In order to validate the accuracy of the CFD results, a test rig as shown in Figure 6 was set up to measure the pump performance. The model pump is connected to the rigid pipes by two rubber hoses. The flow rate is obtained by a LWGY-MIK-DN20 liquid turbine flowmeter with a range of $0.8-8 \mathrm{~m}^{3} / \mathrm{h}$ and accuracy of $0.5 \%$. The head of the pump is measured by a CYG1204F type differential pressure transmitter with an uncertainty of $0.1 \%$ and range of $0-200 \mathrm{kPa}$. The PSW 30-36 programmable DC power is used to drive the pump. The output voltage range of the power is $0-30 \mathrm{~V}$, and the maximum output current is $36 \mathrm{~A}$. The data acquisition system consists of LabVIEW acquisition program and NI6343 data acquisition card. The estimated uncertainty of head and efficiency measurement is below $0.32 \%$, and the random uncertainty is no more than $\pm 0.1 \%$. 


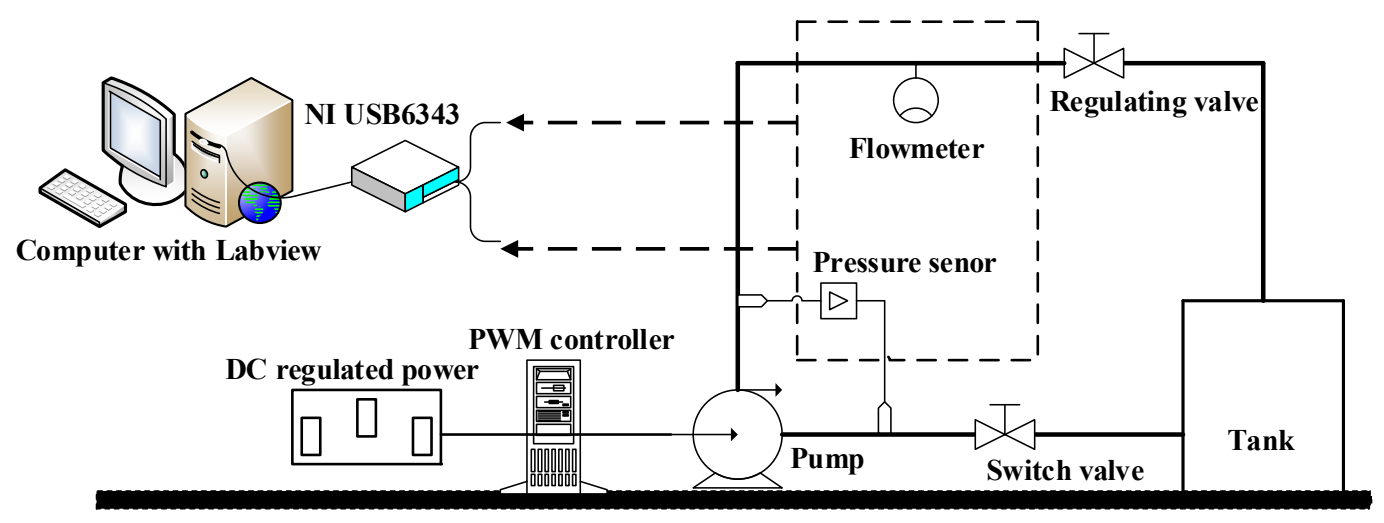

Figure 6. The schematic diagram of test rig.

The obtained pump hydraulic performance curves from CFD and experiment are compared, as shown in Figure 7. The pump efficiency obtained from the test has been converted into the hydraulic one by removing motor efficiency, bearing efficiency, and leakage efficiency. As seen from the results, the numerical ones are always consistent with the changing trends of the experimental curves. Both head and hydraulic efficiency of the simulation results are higher than the experimental values, which might be because the material roughness is not considered in the numerical simulation. The deviations of head at $0.8 Q_{d}, 1.0 Q_{d}, 1.25 Q_{d}$ are $4.9 \%, 4.3 \%, 1.7 \%$, respectively. The deviations of efficiency at $0.8 Q_{d}, 1.0 Q_{d}, 1.25 Q_{d}$ are $3.6 \%, 4.9 \%, 4.8 \%$, respectively. Therefore, it can be stated that the employed numerical method is reliable for the optimization at three selected flowrates.

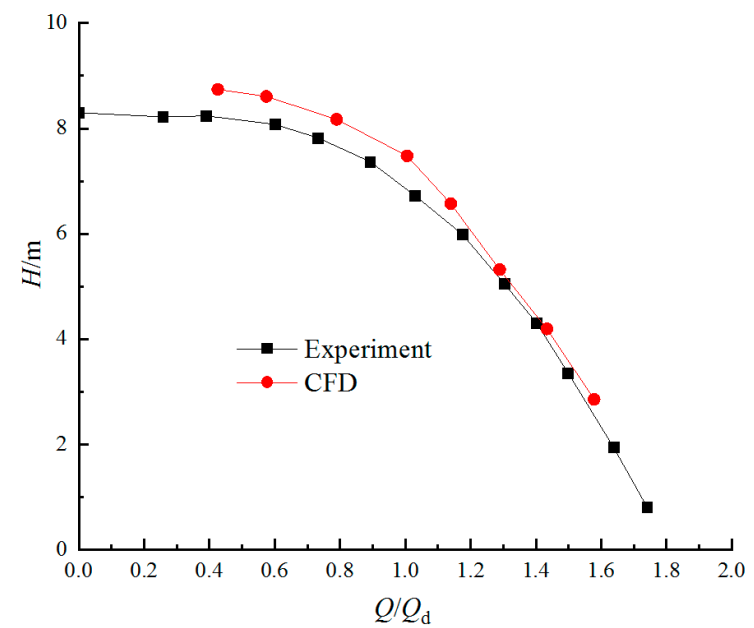

(a)

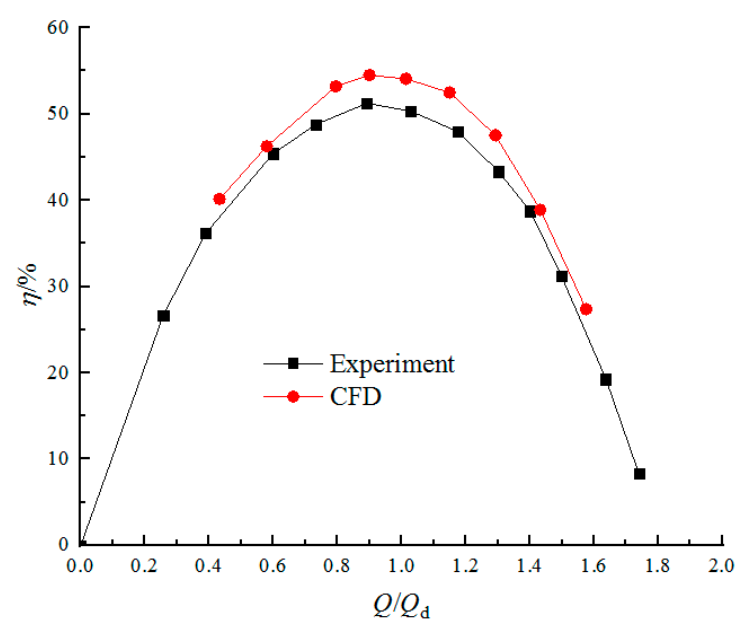

(b)

Figure 7. Comparison of pump performance between numerical and experimental results: (a) Head; (b) Efficiency.

\section{Automatic Intelligent Optimization}

\subsection{Construction of Automatic Optimization Platform}

In order to reduce manual intervention and save optimization time, an automatic optimization platform for CFD is established. The platform integrates a 3D parametric design module, mesh division module, pre-processing module, solver, and post-processing module with computer batch processing commands, which can automatically realize the combination of parametric design, grid generation, pre-process for CFD, simulation, and post-process for CFD. The operation process of this optimization platform is shown in Figure 8. The numerical optimization algorithm scheme is set in the DOE part. 


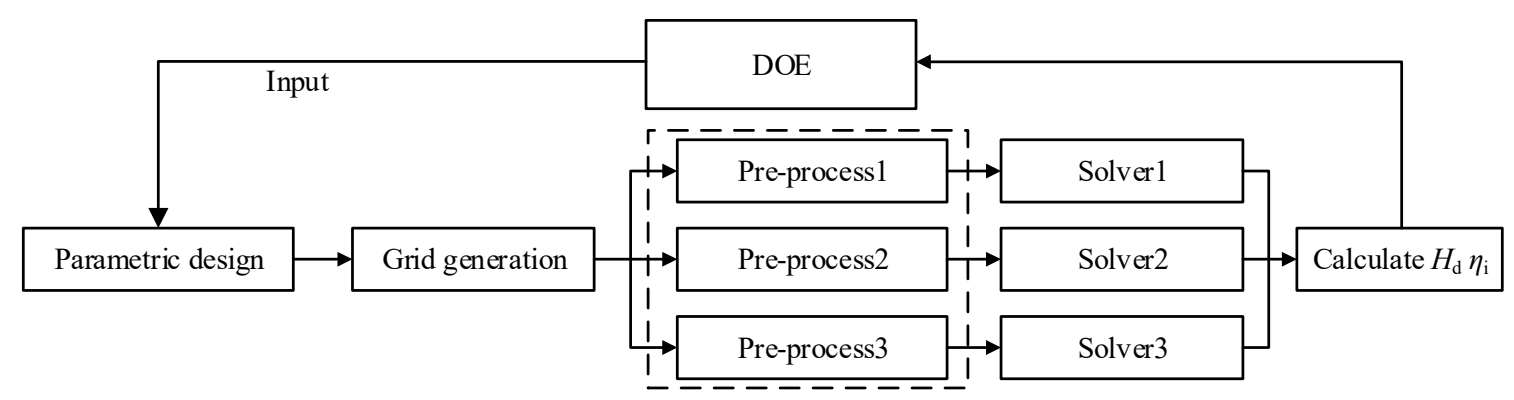

Figure 8. Operation process of the automatic optimization platform.

A computer-aided optimization platform, namely Isight, has been widely used in multidisciplinary design and optimization. Its rich component library and algorithm library, visualization of the running process, and powerful data analysis functions are useful for optimization design in many fields [31]. This study uses this software to realize the integration between 3D modeling software CFturbo and numerical simulation software Ansys CFX14.1. Thus, an intelligent optimization platform for multi-conditions of automotive electronic water pumps is built, as shown in Figure 9.

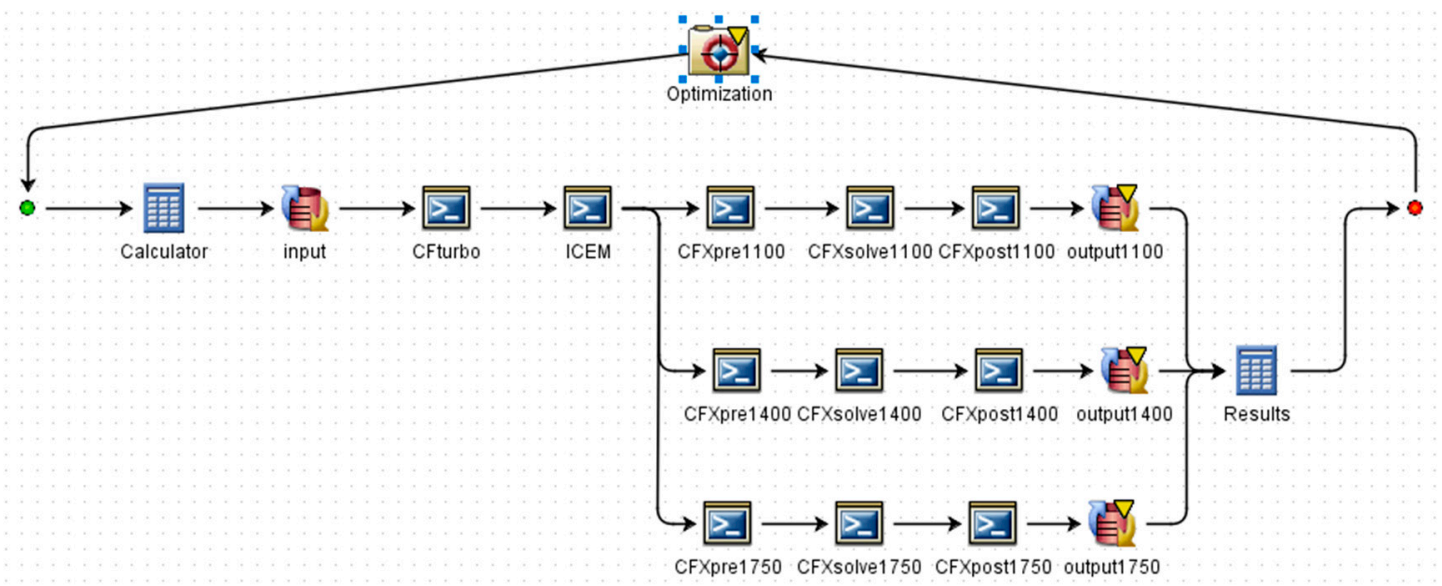

Figure 9. Integrated optimization platform of automotive electronic water pump.

\subsection{Orthogonal Design Optimization}

DOE is one of the most important methods in today's product development and performance optimization. Its function is similar to the mathematical arrangement, which makes a large number of data reasonably and orderly arranged. It provides a scientific experimental scheme for designers. The two main concepts in the experimental design are factors (input variables in the design) and levels (the number of values in each variable). In this study, three levels are selected for each factor, as shown in Table 3.

Table 3. Levels of factors in orthogonal experiments.

\begin{tabular}{ccccc}
\hline \multirow{2}{*}{ Levels } & \multicolumn{4}{c}{ Factors } \\
\cline { 2 - 5 } & $\mathbf{A}$ & $\mathbf{B}$ & $\mathbf{C}$ & $\mathbf{D}$ \\
& $\boldsymbol{D}_{\mathbf{2}} / \mathbf{m m}$ & $\boldsymbol{b}_{\mathbf{2}} / \mathbf{m m}$ & $\mathbf{Z}$ & $\boldsymbol{\beta}_{\mathbf{2}} /\left(^{\circ}\right)$ \\
\hline 1 & 44 & 3 & 4 & 30 \\
2 & 45 & 3.2 & 5 & 33 \\
3 & 46 & 3.5 & 6 & 35 \\
\hline
\end{tabular}

As is listed in Table 4, a pair-wise comparison square matrix was made from the comparison factors based on experience, the diagonal elements of the matrix are always 1 because the same factors have 
the same weightage. The weight value $\left(\omega_{i}\right)$ of each objective is then calculated based on Equation (8). As seen from Table 4, CR is less than 0.1 , which means the values of $\omega_{i}$ listed in the last column can be applied in this multi-objective problem.

Table 4. Evaluation results and weight factors. $C R$, consistency ratio.

\begin{tabular}{ccccc}
\hline Objective & $\eta_{\mathbf{1}}$ & $\eta_{\mathbf{2}}$ & $\eta_{\mathbf{3}}$ & $\omega_{\mathbf{i}}$ \\
\hline$\eta_{1}$ & 1 & $2 / 3$ & $10 / 11$ & 0.2786 \\
$\eta_{2}$ & $3 / 2$ & 1 & $5 / 4$ & 0.4059 \\
$\eta_{3}$ & $11 / 10$ & $4 / 5$ & 1 & 0.3155 \\
$C R$ & & 0.000725 & & \\
\hline
\end{tabular}

Theoretically, there are 81 (calculated by $3^{4}$ ) kinds of schemes, while nine kinds use orthogonal arrays to have the same effect. Orthogonal arrays are compiled into a standardized table using the mathematical "orthogonality" principle to arrange the experimental program scientifically. This method was first applied to engineering design by Plackett and Burman [32]. A notational scheme to characterize the orthogonal table, as Equation (14) represents:

$$
L_{m}\left(n^{p}\right)
$$

where, $n$ is the number of levels; $p$ is the number of factors; $m$ is the number of schemes.

In this study, $L_{9}\left(3^{4}\right)$ orthogonal table is applied to obtain the space-filling samples. Four factors and three levels are reasonably divided into nine groups of experimental schemes. The evaluation indexes results $f(X)$ are shown in Table 5, which are calculated according to Equation (1) based on the CFD method.

Table 5. Test schemes.

\begin{tabular}{|c|c|c|c|c|c|c|c|c|c|}
\hline \multirow{2}{*}{ Number } & \multicolumn{4}{|c|}{ Levels } & \multicolumn{4}{|c|}{ Corresponding Parameters } & \multirow{2}{*}{$\begin{array}{c}\text { Results } \\
f(X)\end{array}$} \\
\hline & A & B & $\mathrm{C}$ & $\mathbf{D}$ & $D_{2}$ & $b_{2}$ & $Z$ & $\beta_{2}$ & \\
\hline 1 & $A_{1}$ & $B_{1}$ & $C_{1}$ & $D_{1}$ & 44 & 3 & 4 & 30 & 46.11 \\
\hline 2 & $A_{1}$ & $B_{2}$ & $C_{2}$ & $D_{2}$ & 44 & 3.2 & 5 & 33 & 50.92 \\
\hline 3 & $A_{1}$ & $B_{3}$ & $C_{3}$ & $D_{3}$ & 44 & 3.5 & 6 & 35 & 50.81 \\
\hline 4 & $A_{2}$ & $B_{1}$ & $C_{2}$ & $D_{3}$ & 45 & 3 & 5 & 35 & 50.89 \\
\hline 5 & $A_{2}$ & $B_{2}$ & $\mathrm{C}_{3}$ & $D_{1}$ & 45 & 3.2 & 6 & 30 & 51.21 \\
\hline 6 & $A_{2}$ & $B_{3}$ & $C_{1}$ & $D_{2}$ & 45 & 3.5 & 4 & 33 & 48.35 \\
\hline 7 & $A_{3}$ & $B_{1}$ & $C_{3}$ & $D_{2}$ & 46 & 3 & 6 & 33 & 51.53 \\
\hline 8 & $A_{3}$ & $B_{2}$ & $C_{1}$ & $D_{3}$ & 46 & 3.2 & 4 & 35 & 49.73 \\
\hline 9 & $A_{3}$ & $B_{3}$ & $C_{2}$ & $D_{1}$ & 46 & 3.5 & 5 & 30 & 51.64 \\
\hline
\end{tabular}

\subsubsection{Range and Sensitivity Analysis}

The range analysis was performed to evaluate the influence of different factors on the evaluation index. The factor with the largest range was considered as the most sensitive factor, which had the greatest impact on the evaluation index. First of all, the comprehensive average value of $t$ at the same level of each factor was obtained by Equation (15) On this basis, the range of each influencing factor was obtained by Equation (16), subtracting the minimum value from the maximum value of $t$ at different levels of each factors. The results are shown in Table 6.

$$
\begin{gathered}
t_{i}=\frac{T_{i}}{r}, \\
R=\max \left(t_{1}, t_{2}, t_{3}\right)-\min \left(t_{1}, t_{2}, t_{3}\right),
\end{gathered}
$$

where $T_{i}$ represents the sum of all test target values at the $i$ level; $r$ is the number of different factor levels; $t_{i}$ denotes the average of the test target values and $R$ is the range. 
Table 6. Range analysis.

\begin{tabular}{ccccc}
\hline \multirow{2}{*}{ Target } & \multicolumn{4}{c}{$f(X) \%$} \\
\cline { 2 - 5 } & $\mathbf{A}$ & $\mathbf{B}$ & $\mathbf{C}$ & $\mathbf{D}$ \\
\hline$t_{1}$ & 49.28 & 49.51 & 48.06 & 49.65 \\
$t_{2}$ & 50.15 & 50.62 & 51.15 & 50.27 \\
$t_{3}$ & 50.97 & 50.27 & 51.18 & 50.48 \\
$R$ & 1.69 & 1.11 & 3.12 & 0.83 \\
\hline
\end{tabular}

In order to visually reflect the impact size and trend of each factor on the evaluation target, the average value and factor level of the test target are drawn as a factors and indicators trend graph, as is shown in Figure 10. As seen from the range value analysis, the order of importance on efficiency is $C$, $\mathrm{A}, \mathrm{B}$, and $\mathrm{D}$. The primary and secondary order of influence of each factor on the target is: $Z>D_{2}>b_{2}$ $>\beta_{2}$. The blade number $Z$ has the greatest impact on pump efficiency, followed by the factor $A$. As the blade number $Z$ and impeller outlet diameter $D_{2}$ increase, the hydraulic performance improves significantly. Generally, the effect of impeller outlet width $b_{2}$ on efficiency is relatively small.

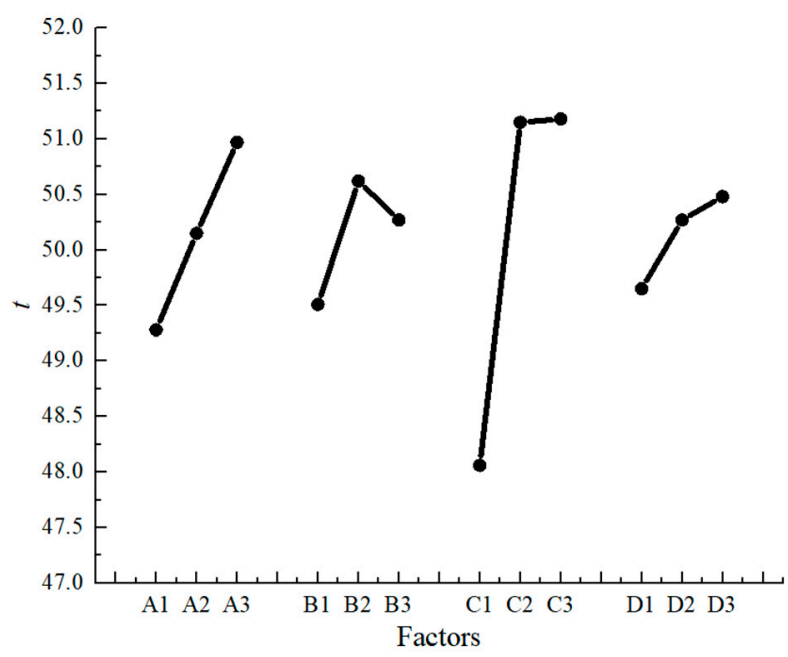

Figure 10. Range analysis diagram.

Analysis of variance (ANOVA) is an effective method to determine the significance of controllable factors on the research results [33]. It tests the variables by mean squaring and estimates experimental errors at specific levels. F-test is always used in ANOVA to analyze whether a particular design has any significant change in quality standards. In analyzing the parameters, the $F$-test tool based on ratio of mean square and residual error is used to find the significance of a factor. It is evaluated by using the equations below.

The sum of squared total deviation $S S_{T}$ and total degrees of freedom $f_{T}$ are defined as:

$$
\begin{gathered}
S S_{T}=\sum_{j=1}^{m} f_{j}^{2}(x)-\frac{T^{2}}{m}(j=1,2, \ldots, m) \\
f_{T}=m-1
\end{gathered}
$$

where $m$ is the number of simples, $m=9$ in this study. $T$ is the sum of the nine scheme results. The sum of square $S S_{k}$ and degrees of freedom of each factor $f_{k}$ are defined as follows:

$$
\begin{gathered}
S S_{k}=\frac{1}{n} \sum_{k=1}^{n} T_{k}^{2}-\frac{T^{2}}{m}(k=1,2, \ldots, n) \\
f_{k}=n-1
\end{gathered}
$$


where $k=1,2,3,4$ represents the factors A, B, C, D, respectively, $n=4$.

The sum of the square $S S_{E}$ and degree of freedom of the deviations $f_{E}$ are defined as follows:

$$
\begin{gathered}
S S_{E}=S S_{T}-\sum_{k=1}^{n} S S_{k} \\
f_{E}=f_{T}-\sum_{k=1}^{n} f_{k}
\end{gathered}
$$

The final $F_{k}$ value for significance level is defined by the Equation (20).

$$
F_{k}=\frac{S S_{k} / f_{k}}{S S_{E} / f_{E}} .
$$

In general, the $F$ value for significance level $\alpha$ (usually $\alpha=0.05$ or $\alpha=0.1$ ) should always be used as quantile points to judge if the factor $k$ plays important role in effecting the results. Values of $F_{\alpha}$ could be obtained from the $F$ distribution table. Comparing $F_{k}$ obtained by calculating the $F$ value to $F_{\alpha}$ from the F-table [32]: if $F_{k}>F_{0.05}$, the factor $k$ is considered to have a highly significant effect on the test result; if $F_{k}>F_{0.1}$, the factor $k$ is considered to have a significant influence on the test result; otherwise, it can be assumed that factor $k$ has little effect on the results. The calculation results of ANOVA for all factors are shown in Table 7. From the common F-test table, $F_{0.05}(2,2)=19$ and $F_{0.1}(2,2)=9$ for the given degree of freedom shown in Table 7.

Table 7. Analysis of variance.

\begin{tabular}{ccccc}
\hline Source of Variance & \multicolumn{5}{c}{$f(X)$ \% } \\
\cline { 2 - 5 } & $\mathbf{A}$ & $\mathbf{B}$ & $\mathbf{C}$ & $\mathbf{D}$ \\
\hline Sum of squared deviation $S S_{T}$ & 4.269 & 1.929 & 19.263 & 1.098 \\
Degree of freedom $f_{T}$ & 2 & 2 & 2 & 2 \\
Mean square error $S S_{E}$ & 1.1 & 1.1 & 1.1 & 1.1 \\
Statistics value $F_{k}$ & 3.888 & 1.757 & 17.544 & 1 \\
Significance & Insignificant & Insignificant & Significant & Insignificant \\
\hline
\end{tabular}

Level $\alpha=0.1$, that is $F_{\alpha}(2,2)=9$, was used to judge the significance of the factor in this study. It can be concluded from Table 7 that the factor $C$ has significant influence to pump performance, and the factors' order of importance is $\mathrm{C}, \mathrm{A}, \mathrm{B}$, and $\mathrm{D}$, which is consistent with the trend of the range analysis results. By comparing the $t_{i}$ value, it can be determined that the superior levels of each factor are A3, B2, C3, and D3. According to the sensitivity analysis, the final optimized combination is A3B2C3D3 (scheme 2), and the specific parameters are impeller outlet diameter $D_{2}=46 \mathrm{~mm}$, blade outlet width $b_{2}=3.2 \mathrm{~mm}$, blade number $Z=6$, blade outlet angle $\beta_{2}=35^{\circ}$. The impeller used this optimization scheme compares with the original scheme, while numerical simulations were carried out using the same CFD process.

\subsubsection{Analysis of Optimization Results}

The comparison results of the optimized scheme 2 with the original scheme 1 is shown in Table 8. As seen from it, the heads of each assessment point are improved after optimization, in detail, the efficiency at design flowrate is increased by $3.66 \%$, and the weighted average efficiency of the optimization scheme is increased by $3.29 \%$.

Figure 11 shows the comparison of the external characteristic curves obtained from the CFD. It is clear that the head of the optimized pump is higher than the model pump, and the head curve is shifted to the direction of large flow. It can be seen from the $\eta-Q$ curve that the flow rate at best efficiency point increased slightly. The efficiency improvement under large flow conditions is very obvious. Thus, the high efficiency region is effectively broadened. 
Table 8. Comparison of the performances under specified conditions.

\begin{tabular}{ccccccc}
\hline \multirow{2}{*}{ Operation Points } & \multicolumn{2}{c}{$H / \mathbf{m}$} & \multicolumn{2}{c}{$\eta / \%$} & \multicolumn{2}{c}{ Weighted Average Efficiency \% } \\
\cline { 2 - 6 } & Original & Optimal & Original & Optimal & Original & Optimal \\
\hline $0.8 Q_{\mathrm{d}}$ & 7.6 & 7.9 & 50.1 & 52.17 & & \\
$1.0 Q_{\mathrm{d}}$ & 6.7 & 7.3 & 51.4 & 55.06 & 49.93 & 53.22 \\
$1.25 Q_{\mathrm{d}}$ & 5.35 & 6.15 & 47.97 & 51.8 & & \\
\hline
\end{tabular}

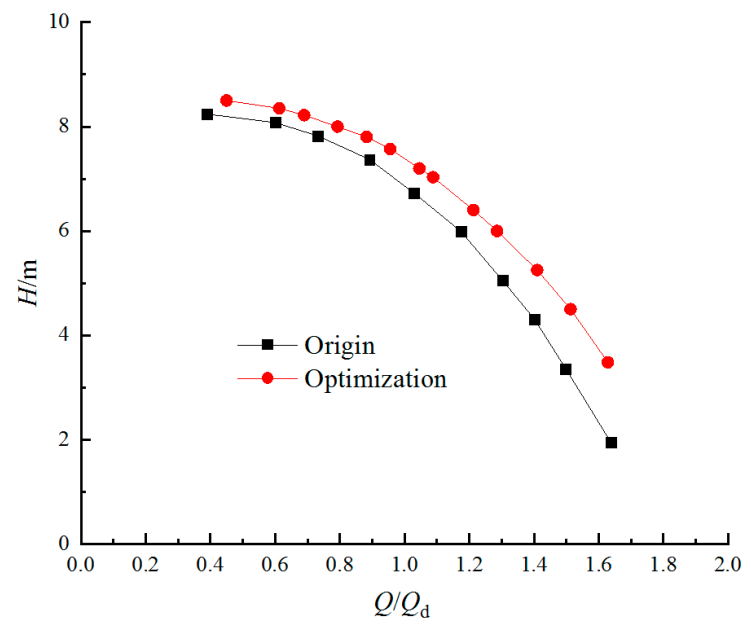

(a)

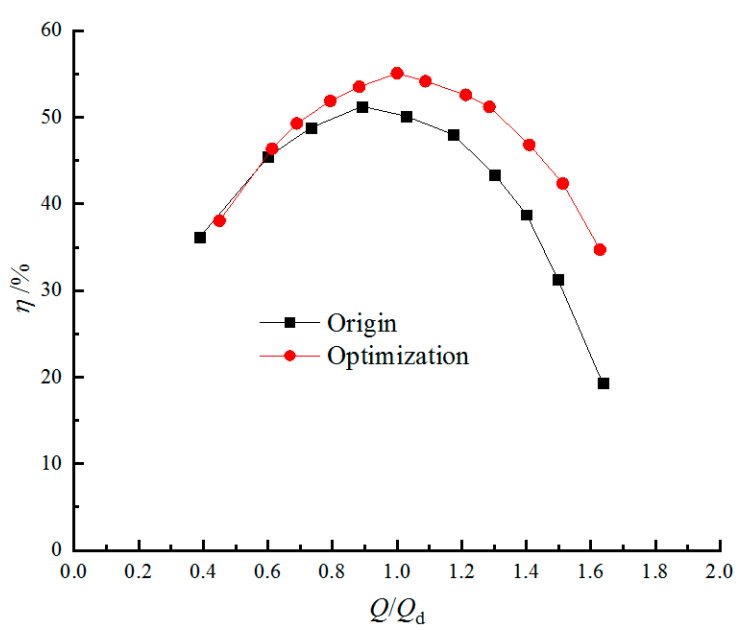

(b)

Figure 11. Comparison of external characteristic curves between origin and optimization scheme: (a) Head; (b) Efficiency.

Figure 12 shows the comparison of pressure distribution at mid-span of the impeller under three flowrates. Figure $12 \mathrm{a}-\mathrm{c}$ represent the results of the original scheme and Figure $12 \mathrm{~d}-\mathrm{f}$ represent the results of the optimized scheme. As seen from them, the pressure inside the impeller passage increases from leading edge to trailing edge under all three different flowrates and appears largest near the impeller outlet. The pressure on the blade working surface is greater than the pressure on the back side of the corresponding position, and the low-pressure area appears on the suction side of the blade near the impeller inlet. As the flow rate increases, the area of the low-pressure area gradually expands. Under $1.25 Q_{d}$, a reverse pressure gradient appears on the inlet part in the optimized impeller. The pressure distribution in the optimized pump impeller is more uniform than in the original one, and a relatively less obvious pressure gradient is present inside the impeller flow-path than the original one.
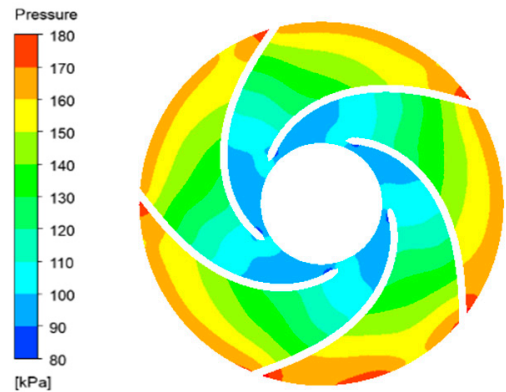

(a)

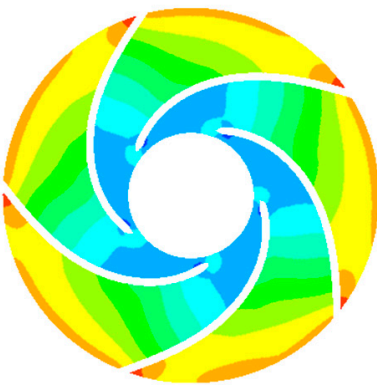

(b)

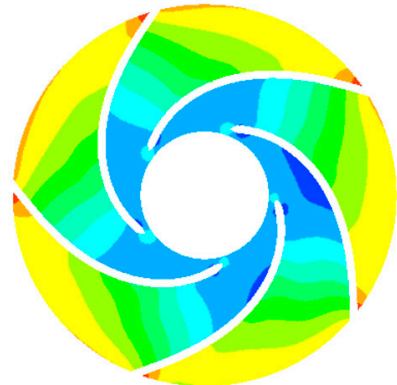

(c)

Figure 12. Cont. 


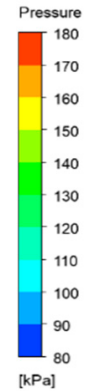

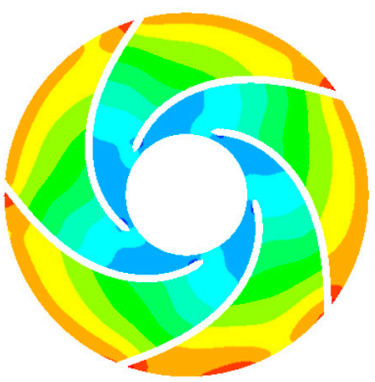

(a)

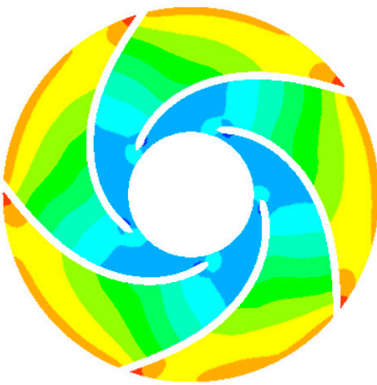

(b)

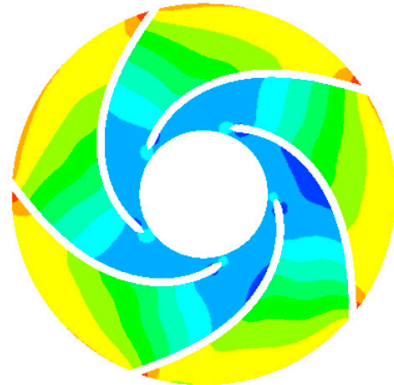

(c)

Figure 12. Comparison of pressure distribution in impeller for the two schemes: (a) origin, $0.8 Q_{d}$; (b) origin, $1.0 Q_{\mathrm{d}} ;(\mathbf{c})$ origin, $1.25 Q_{\mathrm{d}} ;(\mathbf{d})$ optimization, $0.8 Q_{\mathrm{d}} ;(\mathbf{e})$ optimization, $1.0 Q_{\mathrm{d}} ;(\mathbf{f})$ optimization, $1.25 Q_{\mathrm{d}}$.

Figure 13 illustrates the distribution of the turbulence kinetic energy at mid-span of the impeller under three flowrates. Figure $13 \mathrm{a}-\mathrm{c}$ represent the original scheme, Figure $13 \mathrm{~d}-\mathrm{f}$ represent the optimized scheme. It can be concluded that the maximum turbulence kinetic energy is prone to appear on the suction side of the blade. As the flow rate increases, the turbulence kinetic energy in the impeller gradually increases for both schemes. The turbulence kinetic energy of the optimized pump is smaller than the original one. Under $1.0 Q_{d}$, the distribution of turbulence kinetic energy in the flow-path is the most uniform both before and after optimization. Under $1.25 Q_{d}$, the kinetic energy distribution of the optimized pump is more even. Overall, the flow field has been improved after optimization.
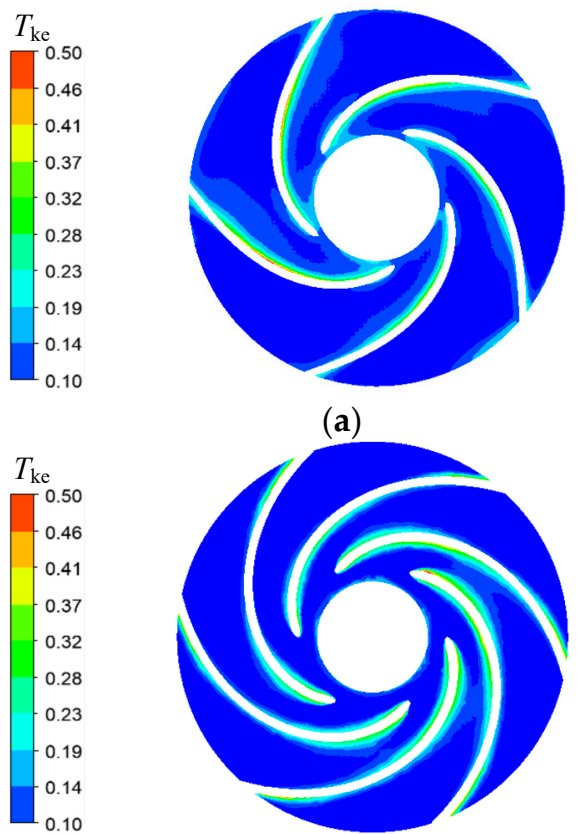

(a)

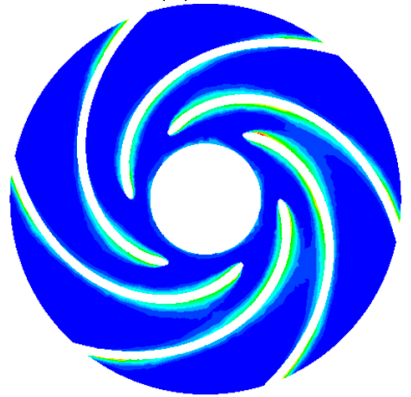

(d)

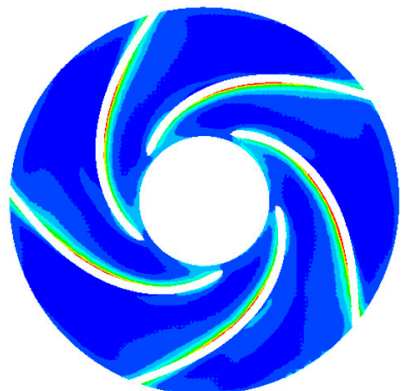

(b)

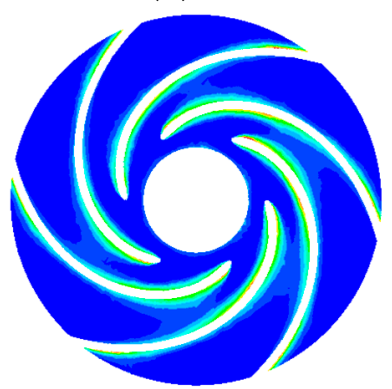

(e)

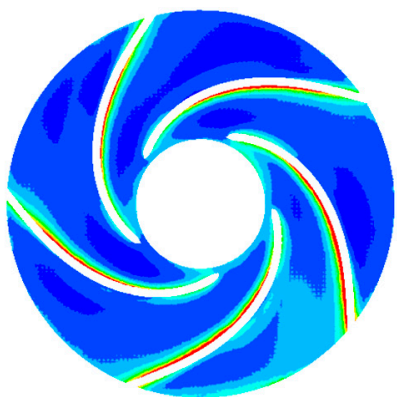

(c)

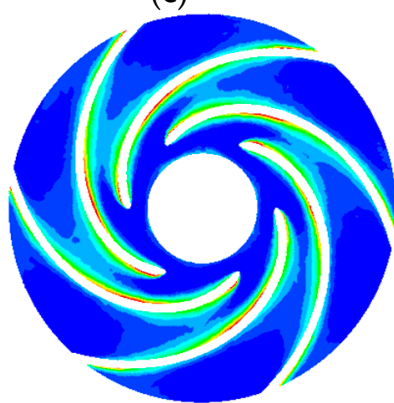

(f)

Figure 13. Comparison of turbulence kinetic energy in impeller for the two schemes: (a) origin, $0.8 Q_{d}$; (b) origin, $1.0 Q_{\mathrm{d}} ;(\mathbf{c})$ origin, $1.25 Q_{\mathrm{d}} ;(\mathrm{d})$ optimization, $0.8 Q_{\mathrm{d}} ;(\mathbf{e})$ optimization, $1.0 Q_{\mathrm{d}} ;(\mathbf{f})$ optimization, $1.25 Q_{\mathrm{d}}$.

Figure 14 shows the comparison of the streamline in the volute at mid-span under three flowrates. Figure $14 \mathrm{a}-\mathrm{c}$ represent the original scheme, Figure $14 \mathrm{~d}-\mathrm{f}$ represent the optimized scheme. It can be concluded that there is obvious flow separation in the volute outlet area accompanied by a large zone with low speed in the original scheme. Under the condition of $1.25 Q_{d}$, a large low-speed vortex region appears in the volute outlet, and the flow state is very disordered, which generates large displacement 
to the mainstream. Under the $0.8 Q_{\mathrm{d}}$ and $1.0 Q_{\mathrm{d}}$ conditions, there are almost no obvious low-speed region and flow separation region in the outlet part of the optimized pump volute, and the flow velocity distribution is relatively uniform, indicating that the flow loss is small. Under $1.25 Q_{d}$, flow separation phenomenon also occurs in the outlet part of the optimized pump. However, the size of the low-speed vortex region in the optimized pump is smaller than the original one, the velocity distribution in the spiral flow-path is more uniform, and the overall flow loss is greatly reduced. The optimized impeller improves the influence of rotor-stator interaction between the impeller and the volute tongue.

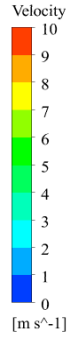

Velocity
\begin{tabular}{|c}
10 \\
-9 \\
-8 \\
-7 \\
6 \\
-5 \\
-4 \\
-3 \\
-2 \\
1 \\
0 \\
{$\left[\mathrm{~ms}^{\wedge}-1\right]$}
\end{tabular}

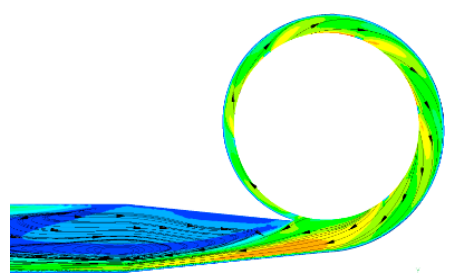

(a)

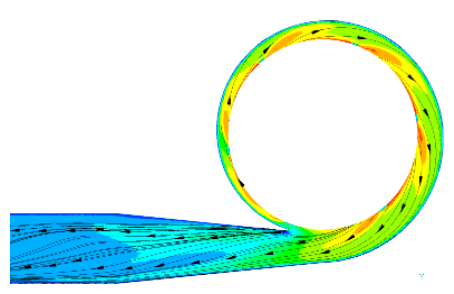

(d)

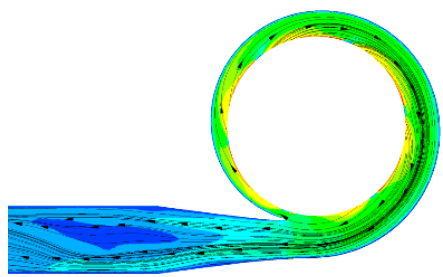

(b)

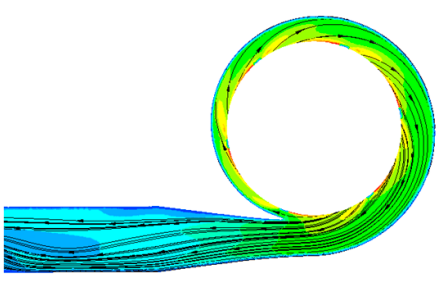

(e)

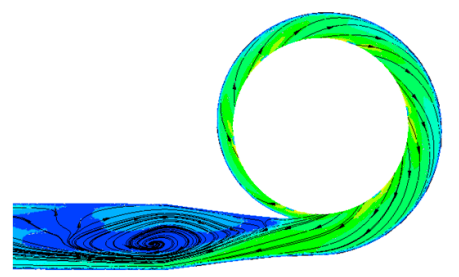

(c)

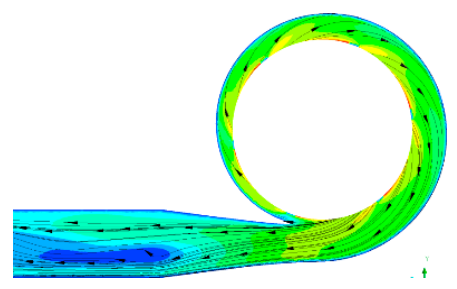

$(\mathbf{f})$

Figure 14. Comparison of velocity streamline in volute for the two schemes: (a) origin, $0.8 Q_{d} ;(\mathbf{b})$ origin, $1.0 Q_{\mathrm{d}} ;(\mathbf{c})$ origin, $1.25 Q_{\mathrm{d}} ;(\mathbf{d})$ optimization, $0.8 Q_{\mathrm{d}} ;(\mathbf{e})$ optimization, $1.0 Q_{\mathrm{d}} ;$ (f) optimization, $1.25 Q_{\mathrm{d}}$.

\subsection{Automatic Optimization with Intelligent Method}

There is a complex multi-peak nonlinear relationship between the optimization objective and geometric parameters. Orthogonal design can only obtain a local optimal solution with fewer variables in discrete space. To further optimize the model pump in overall range quickly and efficiently, an intelligent algorithm program based on an automatic operation platform is needed [34]. Based on the analysis in the previous section, the selected optimization variables and the ranges are shown in Table 9, which includes the impeller diameter $D_{2}$, the width of impeller outlet $b_{2}$, inlet blade angle $\beta_{1}$, outlet blade angle $\beta_{2}$, leading edge tangential angle $t_{3}$, trailing edge tangential angle $\phi$, and blade thickness $\delta$. The variables did not include the parameter of $Z$ at this step because this parameter plays the most important role in influencing the pump performance. Moreover, the $F$ value of $Z$ is much greater than the other three factors from Table 7 , which means $Z$ has significant influence. So, seven optimization variables with limited ranges in Table 9 were chosen to process the next optimization by intelligent method in order to reduce the scheme numbers.

Table 9. Ranges of optimization variables.

\begin{tabular}{cc}
\hline Parameters & Ranges \\
\hline$D_{2}(\mathrm{~mm})$ & $(45,47)$ \\
$b_{2}(\mathrm{~mm})$ & $(2.5,3.5)$ \\
$\beta_{1}\left({ }^{\circ}\right)$ & $(20,30)$ \\
$\beta_{2}\left(^{\circ}\right)$ & $(30,40)$ \\
$t_{3}\left(^{\circ}\right)$ & $(0,25)$ \\
$\phi\left(^{\circ}\right)$ & $(100,120)$ \\
$\delta(\mathrm{mm})$ & $(2,6)$ \\
\hline
\end{tabular}


The genetic algorithm (GA), introduced by John Holland (1971), is a stochastic search technique based on natural selection and natural genetics mechanism to imitate living beings [35]. It has advantages in solving difficult optimization problems with high complexity and undesirable structure, which has been successfully used in fields of production planning and process optimization. The genetic process mainly includes selection, combination of crossover, and mutation. However, the robustness of the GA is not good enough to avoid premature convergence, which will lead to getting a local optimal solution instead of global one. In this study, an improved algorithm named the multi-island genetic algorithm (MIGA) is applied to solve this problem. The main feature of MIGA that distinguishes it from the traditional genetic algorithm is it divides each population of individuals into several subpopulations, namely 'islands'. All operations of the standard GA (selection, crossover, and mutation) are performed on each island separately. The used migration scheme is random ring, which means that the destination subpopulations are randomly chosen at every migration under the constraint that the migration is performed in sequential order between the subpopulations [36]. The structure and process of the MIGA optimization method is illustrated in Figure 15. MIGA selects individuals on each island to regularly migrate into other islands, and then continues with standard GA operations. The migration operation in the multi-island genetic algorithm keeps the diversity of knowledge, improves the chance of including the global optimal solution, and can suppress the occurrence of precocity. In that case, the MIGA is less likely to fall into local optimal than the GA method.

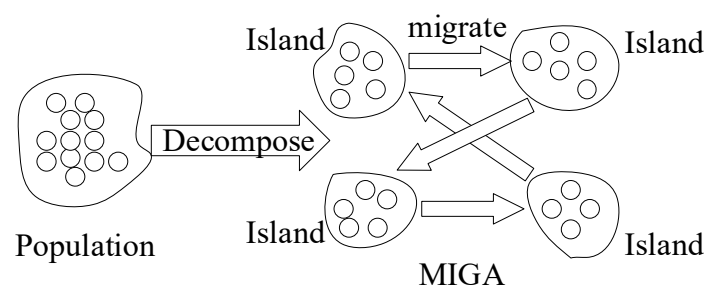

(a)

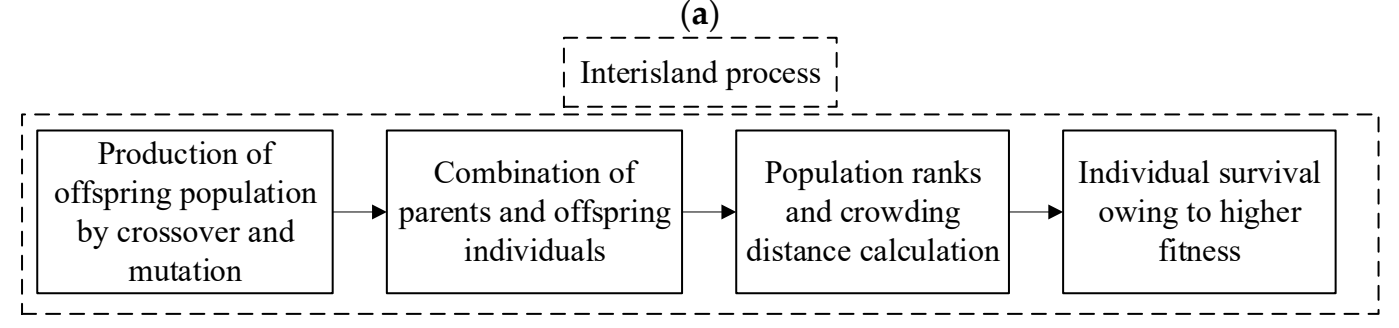

(b)

Figure 15. The structure and process of the multi-island genetic algorithm (MIGA) optimization method: (a) Structure, (b) Process.

Normally, parameters such as subpopulation size, number of islands, generations, crossover rate, migration rate, mutation rate have to be chosen appropriately. The main parameters of the selected MIGA are shown in Table 10. Total population size equal to the result of the subpopulation size multiplied by the number of islands, which is equal to 100 in this study (generally between 20 and 200). Because there are many optimization variables, the number of generations is set to 10, and then the necessary iteration for this optimization is 1000 steps. In order to improve the creating speed of the new individuals, the crossover rate is set to 0.9 , and the mutation rate is set to 0.01 for maintaining the diversity of the population. The migration rate refers to the ratio of individual exchanges between each island, and the default value 0.01 in the software Isight is adopted. The interval of migration is also set to 5 by default. 
Table 10. Optimization parameters adopted in MIGA.

\begin{tabular}{cc}
\hline Parameters & Numerical Value \\
\hline Generations & 10 \\
Subpopulation Size & 10 \\
Number of Islands & 10 \\
Crossover rate & 0.9 \\
Migration rate & 0.01 \\
Interval of Migration & 5 \\
Mutation Rate & 0.01 \\
\hline
\end{tabular}

\subsubsection{Optimization Results}

Optimization based on the MIGA method was processed to obtain the global optimized scheme. Figure 16 shows the variation of the optimization target with the increase of iteration steps. The influence of the optimization variables on the efficiency of the automotive electronic water pump is complicated. The value of the optimization target fluctuates significantly with the increase of the iteration steps, and there are many schemes that do not satisfy the constraint conditions. As the iteration steps increase, the fluctuation value of the target becomes small. It means the multi-island genetic algorithm gradually locates the optimal solution region after a period of searching. The optimal solution (scheme 3) occurred at 798 iteration steps. All the calculations of the optimization works were done by using a normal desktop computer (Dell 7060MT) without running in parallel. The optimized hydraulic scheme was obtained within the limited $375 \mathrm{~h}$.

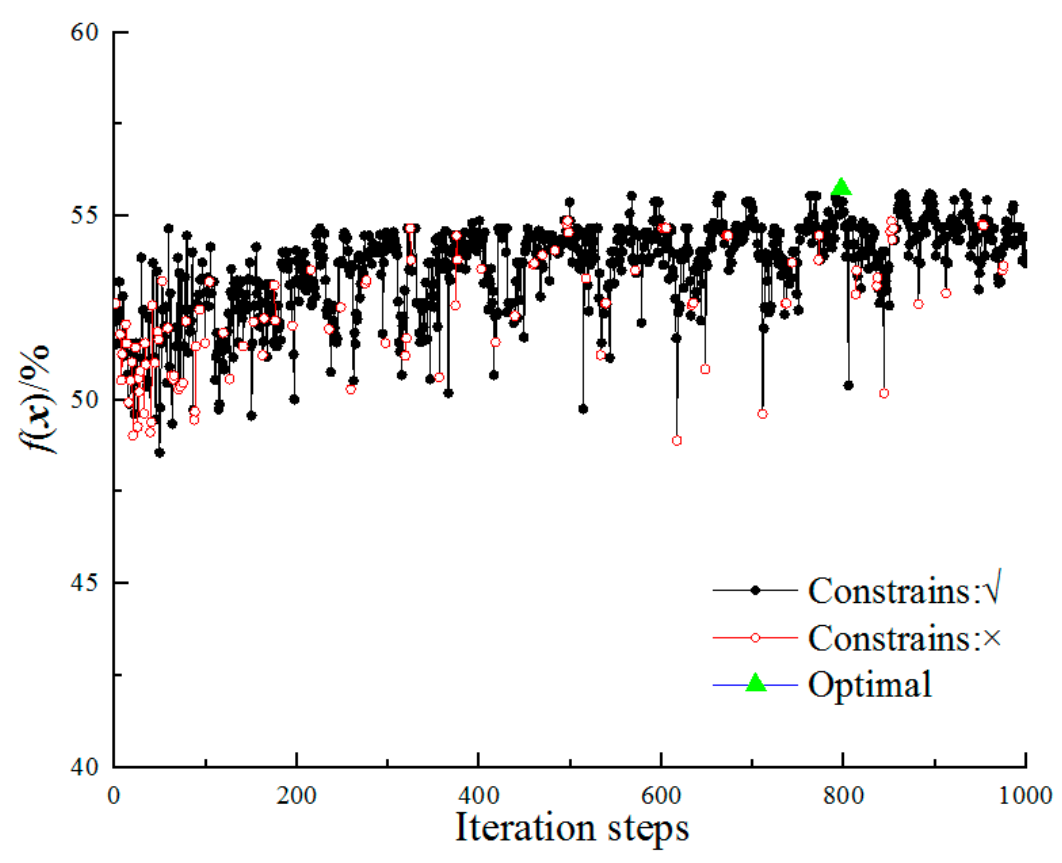

Figure 16. The iteration process of optimization.

Table 11 illustrates the comparison of geometric parameters between scheme 2 and scheme 3 . As seen from the table, the outlet width of scheme 3 increases, the blade outlet angle and wrap angle both decrease slightly, and the impeller diameter becomes smaller. Figure 17 shows a comparison of the three-dimensional model of the two pump impellers. 
Table 11. Comparison of geometric parameters between scheme 2 and scheme 3.

\begin{tabular}{ccccc}
\hline Parameters & Symbols & Unit & Scheme 2 & Scheme 3 \\
\hline Outlet diameter & $D_{2}$ & $\mathrm{~mm}$ & 46 & 45 \\
Outlet width & $b_{2}$ & $\mathrm{~mm}$ & 3.2 & 3.4 \\
Blade number & $Z$ & - & 6 & 6 \\
Blade outlet angle & $\beta_{1}$ & $\circ$ & 22 & 26 \\
Blade outlet angle & $\beta_{2}$ & $\circ$ & 35 & 33 \\
Leading edge tangential angle & $t_{3}$ & $\circ$ & 0 & 8 \\
Trailing edge tangential angle & $\phi$ & $\circ$ & 100 & 115 \\
Blade thickness & $\delta$ & $\mathrm{mm}$ & 2 & 2.4 \\
\hline
\end{tabular}

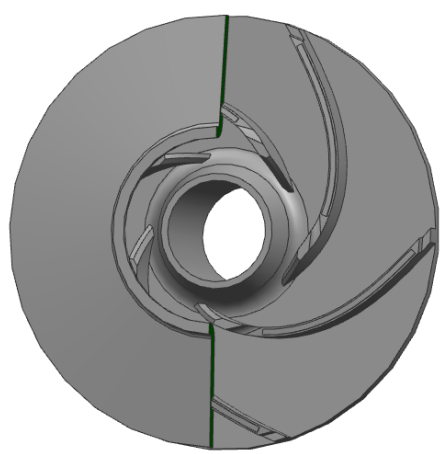

(a)

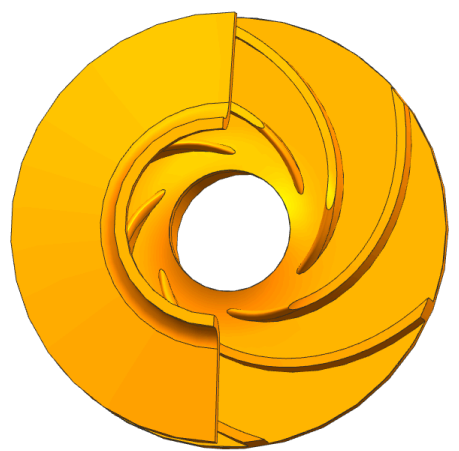

(b)

Figure 17. Comparison of impeller model: (a) scheme 1; (b) scheme 3.

Table 12 shows the head and efficiency of the optimized scheme 3 under three flowrates. It is clear that the head under the design flowrate has significantly improved compared with the original one. The weighted average efficiency under the three flowrates is $55.24 \%$. The maximum efficiency of the pump is increased by $2 \%$, the efficiency of the large flow condition is increased by $3.09 \%$.

Table 12. Performance of optimized scheme (scheme 3).

\begin{tabular}{cccc}
\hline & $\mathbf{0 . 8} \mathbf{Q}_{\mathbf{d}}$ & $\mathbf{1 . 0} \mathbf{Q}_{\mathbf{d}}$ & $\mathbf{1 . 2 5} \boldsymbol{Q}_{\mathbf{d}}$ \\
\hline Head $/ \mathrm{m}$ & 8.57 & 8.15 & 6.86 \\
\hline Efficiency $/ \%$ & 52.98 & 57.06 & 54.89 \\
\hline$F(X) / \%$ & & 55.24 & \\
\hline
\end{tabular}

\subsubsection{Comparison of External Characteristic}

Figure 18 shows the pump performance curve of the automotive electronic water pump obtained from the CFD. As seen from it, the head and efficiency of the optimized pump are obviously improved. The head curve of the optimized scheme 3 is always higher than the original head curve, and the efficiency don't improve much under small flow conditions. However, at the design flowrate, the efficiency is significantly improved, and also under the large flow rate. The efficiency is almost the same for the two schemes under small flow rate. It can be seen that the region with higher efficient working condition of the pump has been broadened for scheme 3 , which means the area with high efficiency value located on the $Q-\eta$ curve is further broadened after optimization. 


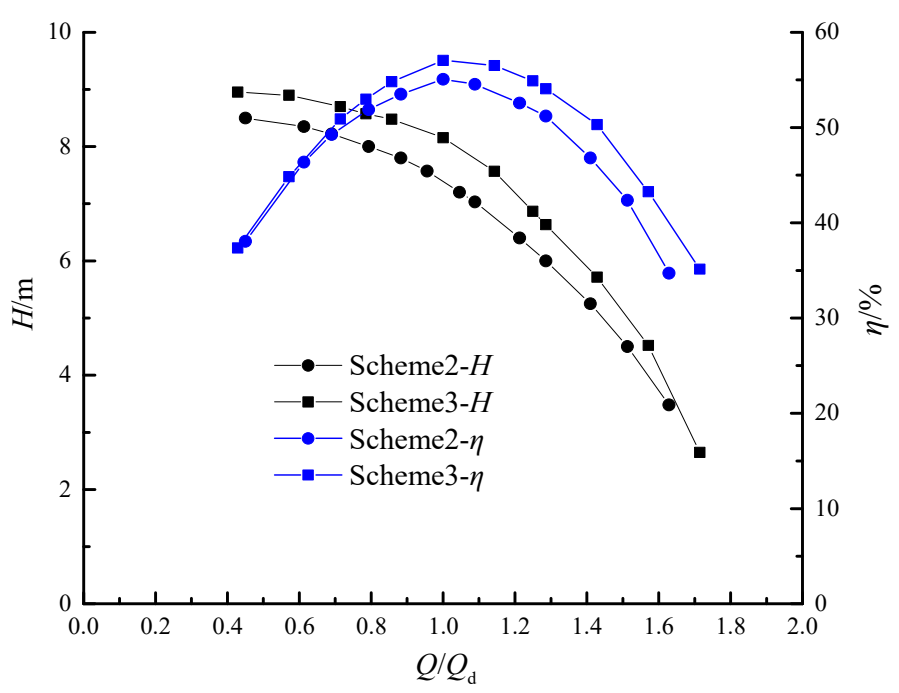

Figure 18. Comparison of external characteristic curves.

\section{Optimization Verification}

\subsection{Comparison of Internal Flow Field}

In this section, the flow characteristics of the cylindrical unfolding surface at different spans of the impeller are compared between the original scheme 1 and optimal scheme 3 . The dimensionless distance of the impeller blade from hub to shroud is defined as:

$$
\text { span }=\frac{r-r_{h}}{r_{t}-r_{h}} \times 100 \%,
$$

where $r_{t}$ is the radius of shroud; $r_{h}$ is the radius of hub; $r$ is the radius of the cylindrical surface.

Figure 19 shows the comparison of velocity streamline at different spans under the design flowrate. It is obvious that a large area of flow separation occurs in the flow passages for scheme 1, large-scale separation vortices appear in the suction side, and the flow state becomes disordered, which seriously affects the performance of the pump. After optimization, the flow in the impeller passage shows a uniform state. The large-scale separation vortex disappears in the flow passages. Thus, the flow loss is reduced, which improves the performance of the pump.

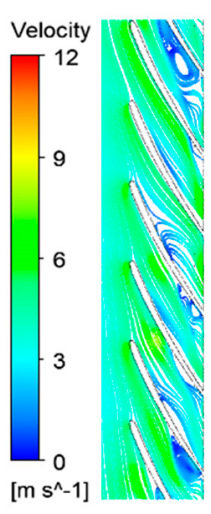

(a)

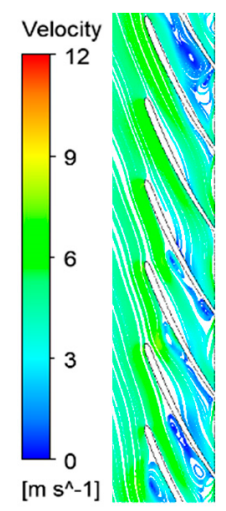

(b)

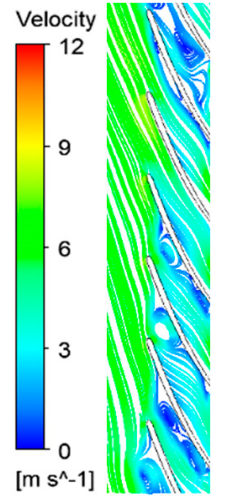

(c)

Figure 19. Cont. 


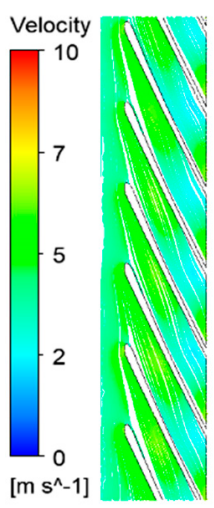

(d)

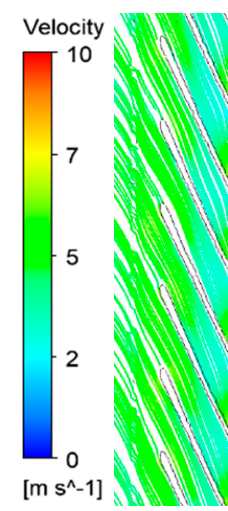

(e)

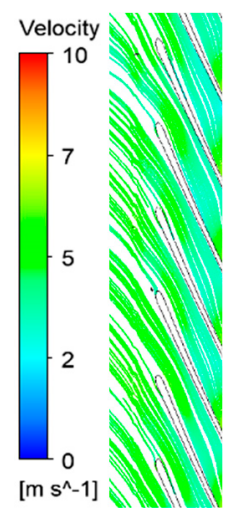

(f)

Figure 19. Comparison of streamline at different spans under $1.0 Q_{\mathrm{d}}$ : (a) Origin scheme 1 , span $=10 \%$; (b) Origin scheme 1, span $=50 \%$; (c) Origin scheme 1, span $=90 \%$; (d) Optimal scheme 3, span $=10 \%$; (e) Optimal scheme 3, span $=50 \%$; (f) Optimal scheme 3, span $=90 \%$.

Figure 20 shows the comparison of the pressure distribution at span $=50 \%$ under three flowrates. From the graph, the pressure distribution of the blade is lower at the wheel hub, the pressure at trailing edge location is higher. As the flow rate increases, the static pressure decreases. Under the same flow rate, the optimized pressure distribution diagram becomes more uniform than that before optimization. The pressure is gradually increased from the wheel hub to the rim, and the pressure at the front edge of the blade is effectively reduced.

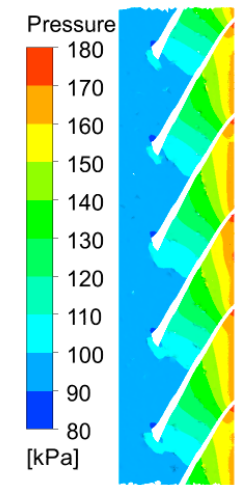

(a)

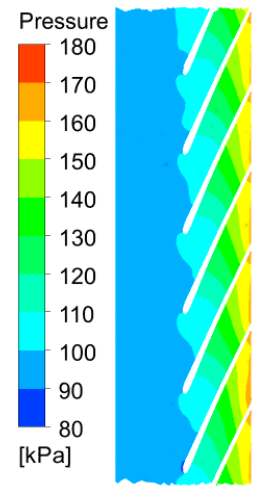

(d)

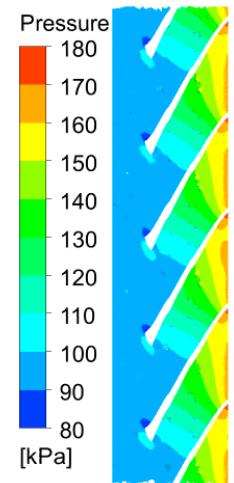

(b)

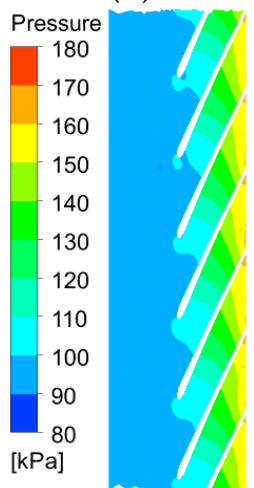

(e)

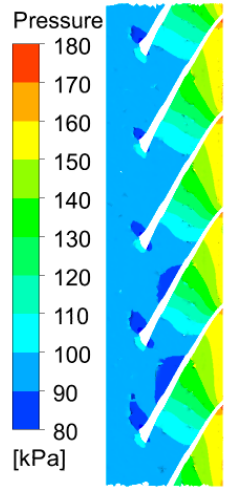

(c)

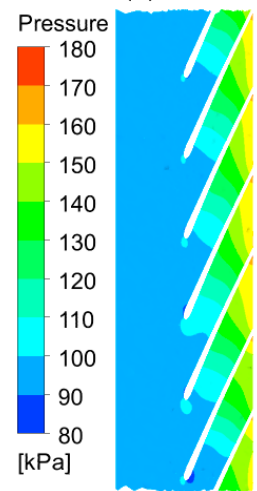

(f)

Figure 20. Pressure comparison at span $=90 \%$ under different working conditions: (a) Origin, $0.8 Q_{d}$; (b) Origin,1.0Q $Q_{\mathrm{d}} ;(\mathbf{c})$ Origin, 1.25 $Q_{\mathrm{d}} ;(\mathbf{d})$ Optimal, $0.8 Q_{\mathrm{d}} ;(\mathbf{e})$ Optimal, 1.0 $Q_{\mathrm{d}} ;(\mathbf{f})$ Optimal, 1.25 $Q_{\mathrm{d}}$.

From the above analysis, the optimization scheme is superior to the original scheme in terms of internal flow. 


\subsection{Test Verification}

In order to verify the reliability of the optimization results, the optimized impeller model (scheme 3) was $3 D$ printed into a prototype for pump characteristic measurement. The test and the pump performance result curves compared with the original scheme are shown in Figure 21.

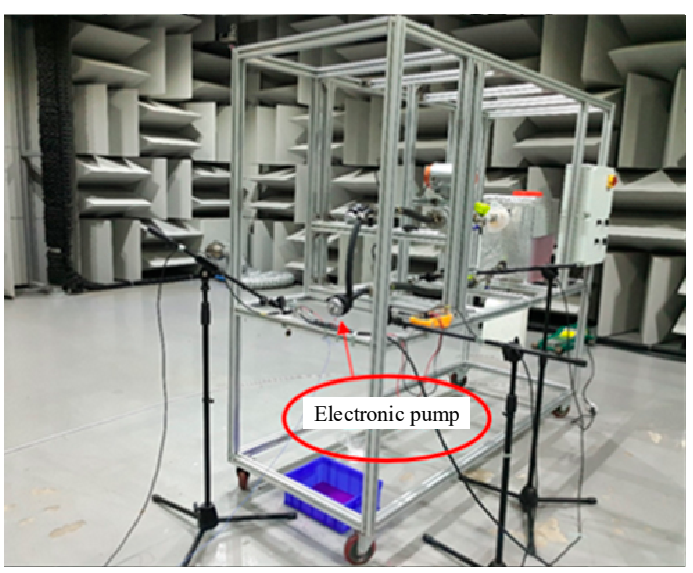

(a)

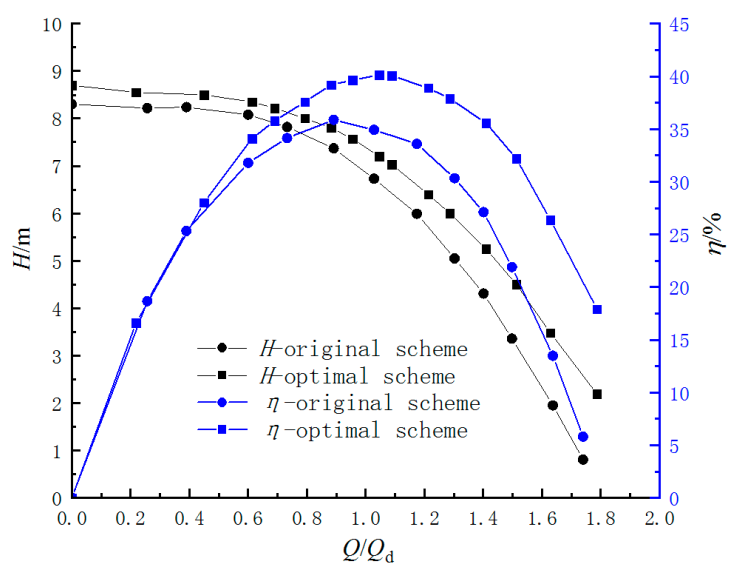

(b)

Figure 21. Comparison of the origin and optimal scheme. (a) Test rig; (b) Comparison pump performance results.

The efficiency obtained by the test of the automobile electronic water pump is the total efficiency of the pump system. As seen from Figure 21b, it is obvious that the head curve of the optimal scheme 3 is basically higher than the original scheme 1 , and the downward trend is slower. The efficiency of the optimization is significantly improved by $4.3 \%$. The efficiency curve of optimal scheme 3 declines slowly under a large flow rate, and the working range with high efficiency of the pump is broadened. The test results are basically consistent with the numerical simulation results, indicating that the optimization method in this study can be applied to the hydraulic optimization design of automotive electronic pumps.

\section{Conclusions}

A multi-point design process based on CFD and intelligent optimization method is proposed in this study to improve the energy transfer efficiency, taking the application of an automotive electronic pump as an example. Firstly, the three-dimensional CFD analysis of the prototype is carried out to understand the flow loss mechanism inside the pump and establish the numerical prediction model of pump performance. Secondly, an automatic optimization platform including fluid domain modeling, meshing, solving, post-processing, and design of experiment (DOE) is built based on the three-dimensional parametric design method. Then, orthogonal experimental design and the multi-island genetic algorithm (MIGA) were utilized to drive the platform for improving the efficiency of the pump at three flowrates. Finally, the optimal impeller geometries were obtained and manufactured into a prototype for verification. The conclusions are as follows.

(1) After orthogonal optimization, the heads of each working point are significantly improved. The weighted average efficiency of the optimization scheme 2 is improved by $3.29 \%$. The number of impeller blades has the most important effects on pump performance improvement. After the intelligent optimization, the high-efficiency region of the automotive electronic water pump is further widened. The efficiency weighted average recorded $55.24 \%$ under three working conditions. The optimal efficiency at design flowrate is increased by $4.3 \%$ after optimization from the experiment test.

(2) There is almost no obvious low-speed region under the optimal conditions and small flow conditions after orthogonal optimization. The flow loss is greatly reduced in the impeller and volute flow-path, the number of separation vortices and their area under a large flowrate are 
smaller than the original model pump. The pressure distribution in the impeller flow-path of the optimized pump is more uniform, and the pressure gradient becomes smaller inside the impeller flow-path. the overall turbulence energy is significantly reduced after optimization. All of the above is the root cause of the efficiency improvement.

(3) The automatic optimization platform built in this study combined with intelligent MIGA algorithm could obtain the global optimization scheme among the selected parameter range. The hydraulic performance of automotive electronic water pumps under three multiple operating conditions have significant improvement, realizing the integration of "design-simulation-optimization" in limited time, which means this method has great potential in the application of fluid machinery design.

Author Contributions: Methodology, Q.S. and J.Y.; simulation, G.S.; analyzed the data, R.L. and C.S.; writing-review and editing, Q.S.; funding acquisition and validation, S.X. All authors have read and agreed to the published version of the manuscript.

Funding: This research was funded by National Key Research and Development Program of China (2018YFB0606103), National Natural Foundation of China $(51976079,51779107)$ and China Postdoctoral Science Foundation (2019M661745).

Acknowledgments: The authors gratefully acknowledge the financial support by National Key Research and Development Program of China (2018YFB0606103), National Natural Foundation of China $(51976079,51779107)$ and China Postdoctoral Science Foundation (2019M661745).

Conflicts of Interest: The authors declare no conflict of interest.

\section{References}

1. Shankar, V.K.; Umashankar, S.; Paramasivam, S.; Hanigovszki, N. A comprehensive review on energy efficiency enhancement initiatives in centrifugal pumping system. Appl. Energy 2016, 181, 495-513. [CrossRef]

2. Vlad, H.; Alin, I.B.; Sébastien, L.; Cécile, M.A. A Dynamic Approach for Faster Performance Measurements on Hydraulic Turbomachinery Model Testing. Appl. Sci. 2018, 8, 1426. [CrossRef]

3. Cortona, E.; Onder, C. Engine thermal management with electric cooling pump. SAE Tech. Pap. Ser. 2000, 1, 0965. [CrossRef]

4. Hoon, C.; Dohoy, J.; Zoran, S.F.; Dennis, N.A.; John, V.; Walter, B. Application of controllable electric coolant pump for fuel economy and cooling performance improvement. J. Eng. Gas Turbines Power 2007, 129, 43-50.

5. Wang, X.; Liang, X.; Hao, Z.; Chen, R. Comparison of electrical and mechanical water pump performance in internal combustion engine. Int. J. Veh. Syst. Model. Test. 2015, 10, 205-223. [CrossRef]

6. Si, Q.R.; Yuan, S.Q.; Yuan, J.P.; Wang, C.; Lu, W.G. Multi-objective optimization of low-specific-speed multistage pumps by using matrix analysis and CFD method. J. Appl. Math. 2013, 10, 261-274.

7. Kim, K.Y.; Samad, A.; Benini, E. Design Optimization of Fluid Machinery-Applying Computational Fluid Dynamics and Numerical Optimization; John Wiley \& Sons, Inc.: Hoboken, NJ, USA, 2019.

8. Wang, C.; He, X.; Shi, W.; Wang, X.; Wang, X.; Qiu, N. Numerical study on pressure fluctuation of a multistage centrifugal pump based on whole flow field. AIP Adv. 2019, 9, 035118. [CrossRef]

9. Kim, J.H.; Lee, H.C.; Kim, J.H.; Choi, Y.S.; Yoon, J.Y.; Yoo, I.S.; Choi, W.C. Improvement of hydrodynamic performance of a multiphase pump using design of experiment techniques. J. Fluids Eng. Trans. ASME 2015, 137, 081301. [CrossRef]

10. Wu, D.; Yan, P.; Chen, X. Effect of trailing-edge modification of a mixed-flow pump. J. Fluids Eng. Trans. ASME 2015, 137, 1-9. [CrossRef]

11. Zhou, L.; Shi, W.; Lu, W. Numerical investigations and performance experiments of a deep-well centrifugal pump with different diffusers. J. Fluids Eng. Trans. ASME 2012, 134, 071102. [CrossRef]

12. Osman, M.K.; Wang, W.; Yuan, J.; Zhao, J.; Wang, Y.; Liu, J. Flow loss analysis of a two-stage axially split centrifugal pump with double inlet under different channel designs. Proc. Inst. Mech. Eng. C J. Mech. Eng. Sci. 2019, 233, 5316-5328. [CrossRef]

13. Wang, C.; Shi, W.; Wang, X. Optimal design of multistage centrifugal pump based on the combined energy loss model and computational fluid dynamics. Appl. Energy 2017, 187, 10-26. [CrossRef]

14. Liu, M.; Tan, L.; Cao, S. Design method of controllable blade angle and orthogonal optimization of pressure rise for a multiphase pump. Energies 2018, 11, 1048. [CrossRef] 
15. Li, X.; Liu, Z.; Lin, Y. Multipoint and multiobjective optimization of a centrifugal compressor impeller based on genetic algorithm. Math. Probl. Eng. 2017, 2017,1-18. [CrossRef]

16. Luo, W.; Lyu, W. An application of multidisciplinary design optimization to the hydrodynamic performances of underwater robots. Ocean Eng. 2015, 104, 686-697. [CrossRef]

17. Aziz, M.; Tayarani, N.M.H. An adaptive memetic particle swarm optimization algorithm for finding large-scale latin hypercube designs. Eng. Appl. Artif. Intell. 2014, 36, 222-237. [CrossRef]

18. Michalewicz, Z.; Krawczyk, J.B.; Kazemi, M. Genetic Algorithms and Optimal Control Problem. In Proceedings of the 29th IEEE Conference on Decision and Control, San Francisco, CA, USA, 5-7 December 1990.

19. Pei, J.; Wang, W.; Yuan, S. Multi-point optimization on meridional shape of a centrifugal pump impeller for performance improvement. J. Mech. Sci. Technol. 2016, 30, 4949-4960. [CrossRef]

20. Pei, J.; Wang, W.; Osman, M.K.; Gan, X. Multiparameter optimization for the nonlinear performance improvement of centrifugal pumps using a multilayer neural network. J. Mech. Sci. Technol. 2019, 33, 2681-2691. [CrossRef]

21. Yuan, S.Q.; Wang, W.J.; Pei, J.; Zhang, J.F.; Mao, J.Y. Multi-objective optimization of low-specific-speed centrifugal pump. Trans. Chin. Soc. Agric. Eng. 2015, 31, 46-52.

22. Zhang, Y.; Hu, S.; Wu, J.; Zhang, Y.; Chen, L. Multi-objective optimization of double suction centrifugal pump using Kriging metamodels. Adv. Eng. Softw. 2014, 74, 16-26. [CrossRef]

23. Wang, C.; Ye, J.; Zeng, C.; Xia, Y.; Luo, B. Multi-objective optimum design of high specific speed mixed-flow pump based on NSGA-II genetic algorithm. Trans. Chin. Soc. Agric. Eng. 2015, 31, 100-106.

24. Zhang, J.; Zhu, H.; Li, Y. Shape Optimization of Helico-Axial Multiphase Pump Impeller Based on Genetic Algorithm. In Proceedings of the 2009 Fifth International Conference on Natural Computation, Tianjin, China, 14-16 August 2009.

25. Yang, X.S. Firefly algorithms for multimodal optimization. In Stochastic Algorithms: Foundations and Applications, SAGA 2009, Lecture Notes in Computer Sciences; Springer: Berlin/Heidelberg, Germany, 2009; Volume 5792, pp. 169-178.

26. Shao, Y.; Ou, H.; Guo, P.; Yang, H. Shape optimization of preform tools in forging of aerofoil using a metamodel-assisted multi-island genetic algorithm. J. Chin. Inst. Eng. 2019, 42, 297-308. [CrossRef]

27. Wang, Q.X.; Wang, H.; Qi, Z.Q. An application of nonlinear fuzzy analytic hierarchy process in safety evaluation of coal mine. Saf. Sci. 2016, 86, 78-87. [CrossRef]

28. Ruiz-Padillo, A.; Torija, A.J.; Ramos-Ridao, A.F.; Ruiz, D.P. Application of the fuzzy analytic hierarchy process in multi-criteria decision in noise action plans: Prioritizing road stretches. Environ. Model. Softw. 2016, 81, 45-55. [CrossRef]

29. Chaudhary, P.; Chhetri, S.K.; Joshi, K.M. Application of an analytic hierarchy process (AHP) in the GIS interface for suitable fire site selection: A case study from kathmandu metropolitan city, Nepal. Socio Econ. Plan. Sci. 2016, 53, 60-71. [CrossRef]

30. Zhang, J.; Fu, S. An efficient approach for quantifying parameter uncertainty in the SST turbulence model. Comput. Fluids 2019, 181, 173-187. [CrossRef]

31. Wang, W.; Mo, R.; Zhang, Y. Multi-objective aerodynamic optimization design method of compressor rotor based on Isight. Procedia Eng. 2011, 15, 3699-3703. [CrossRef]

32. Kim, J.H.; Choi, J.H.; Husain, A.; Kim, K.Y. Performance enhancement of axial fan blade through multi-objective optimization techniques. J. Mech. Sci. Technol. 2010, 24, 2059-2066. [CrossRef]

33. Balaram, N.A.; Chennakeshava, R.A. Optimization of tensile strength in TIG welding using TAGUCHI method and analysis of variance (ANOVA). Therm. Sci. Eng. Prog. 2018, 8, 327-339. [CrossRef]

34. Hu, X.; Chen, X.; Zhao, Y.; Zhao, Y. Optimization design of satellite separation systems based on Multi-Island Genetic Algorithm. Adv. Space Res. 2014, 53, 870-876. [CrossRef]

35. Sinha, A.; Malo, P.; Deb, K. Evolutionary algorithm for bilevel optimization using approximations of the lower level optimal solution mapping. Eur. J. Oper. Res. 2016, 257, 395-411. [CrossRef]

36. Wang, W.J.; Yuan, S.Q.; Pei, J.; Zhang, J.F. Optimization of the diffuser in a centrifugal pump by combining response surface method with multi-island genetic algorithm. Proc. IMechE Part. E J. Process. Mech. Eng. 2015, 231, 191-201. [CrossRef]

(C) 2020 by the authors. Licensee MDPI, Basel, Switzerland. This article is an open access article distributed under the terms and conditions of the Creative Commons Attribution (CC BY) license (http://creativecommons.org/licenses/by/4.0/). 